\title{
Angekommen in postmigrantischen Stadtgesellschaften? Eine Annäherung an subjektive Integrationsvorstellungen von Geflüchteten und beruflich oder ehrenamtlich in der Flüchtlingsbetreuung Tätigen
}

\author{
Günther Weiss ${ }^{1}$, Francesca Adam ${ }^{2}$, Stefanie Föbker ${ }^{3}$, Daniela Imani ${ }^{3}$, Carmella Pfaffenbach ${ }^{2}$, and \\ Claus-Christian Wiegandt ${ }^{3}$ \\ ${ }^{1}$ Institut für Geographiedidaktik, Universität zu Köln, Köln, Germany \\ ${ }^{2}$ Geographisches Institut, Rheinisch-Westfälische Technische Hochschule (RWTH) Aachen, Aachen, Germany \\ ${ }^{3}$ Geographisches Institut, Rheinische Friedrich-Wilhelms-Universität Bonn, Bonn, Germany
}

Correspondence: Günther Weiss (g.weiss@uni-koeln.de)

Received: 13 July 2018 - Revised: 12 April 2019 - Accepted: 3 May 2019 - Published: 17 June 2019

\section{Einleitung}

Die Zuwanderung von Geflüchteten stellt, besonders seit der Zuwanderungswelle 2015/16, die deutsche Gesellschaft vor große Herausforderungen. Die öffentliche Debatte zwischen Willkommenskultur und Überfremdungsängsten ist dabei stark ideologisch aufgeladen. Ein gewichtiger Teil der politischen Diskussion wird dort, neben Strategien einer Regulierung der Fluchtmigration, um das Verständnis von ,Integration“ geführt. Hier stehen der traditionellen Vorstellung einer „Leitkultur“ des Herkunftslandes, welcher sich die Zuwanderer anzupassen haben, eher progressivere Ideen gegenüber, die betonen, dass beide Seiten auf Basis gegenseitiger Wertschätzung aufeinander zugehen müssen (Höcke und Schnur, 2016). Mit dem Begriff des „Postmigrantischen“ wird darüber hinausgehend u.a. die grundlegende politische Anerkennung migrationsgesellschaftlicher Realitäten in den Mittelpunkt gerückt, die zwar diskutiert, reguliert und ausgehandelt, aber nicht rückgängig gemacht werden können (Foroutan, 2015:2).

Vor dem Hintergrund dieser Debatten wird in dem vorliegenden Artikel der Frage nachgegangen, welche Vorstellungen auf der einen Seite die FluchtmigrantInnen selbst über ihre „Integration“ in Deutschland haben und welche Vorstellungen bei den Personen vorliegen, welche beruflich oder ehrenamtlich mit der Betreuung neu zugewanderter Geflüchteter zu tun haben. Diese Gegenüberstellung ist in mehrfacher Hinsicht von Interesse: Die Vorstellungen der FluchtmigrantInnen können darüber Aufschluss geben, ob Vorbe- halte existieren oder Barrieren wahrgenommen werden, die verhindern, sich in Deutschland heimisch fühlen zu können. Die Bedürfnisse und Ideen der Zuwanderer über ein gelungenes Einleben können wiederum zur Orientierung für die deutsche Integrationspolitik dienen und auch die Strategien der HelferInnen, die im unmittelbaren Kontakt mit den Geflüchteten stehen, kalibrieren. Erkenntnisse über die Sichtweisen der HelferInnen können demgegenüber erhellen, mit welchen Ansprüchen an eine zu erbringende „Integrationsleistung“, ob unmittelbar ausgesprochen oder eher subtil angedeutet, die Geflüchteten konfrontiert werden. Im Hinblick auf die Perspektive einer Transformation Deutschlands in eine postmigrantische Gesellschaft, die von traditionellen Integrationskonzepten Abschied nimmt (Foroutan, 2015), kann die Untersuchung helfen zu klären, inwieweit sich besonders die Protagonisten der lokalen Unterstützung für Geflüchtete in Deutschland, aber auch die Geflüchteten selbst in ihren Strategien und Tätigkeiten im Alltag einem postmigrantischen Verständnis annähern.

Aus einer postmigrantischen Perspektive auf Gesellschaft kann das Untersuchungsdesign mit Unterscheidung von zwei Gruppen von GesprächspartnerInnen zunächst problematisch erscheinen, da es die Dichotomie von Zugewanderten und Einheimischen zu reproduzieren und zu verfestigen scheint. Mit Blick auf gesellschaftliche Beziehungs- und Machtkonstellationen ist diese Gegenüberstellung allerdings zentral. Denn sie erfolgte nicht unter ethnisch-kulturellen Vorannahmen, sondern unter funktionalen Gesichtspunkten: Geflüch- 
tete stellen eine hinsichtlich gesellschaftlicher Handlungsmöglichkeiten und Positionen besondere, in der Regel besonders marginalisierte Gruppe innerhalb der Gesellschaft dar. Auf der anderen Seite repräsentieren die in der Betreuung von Geflüchteten aktiven Personen die Aufnahmegesellschaft zunächst funktional in ihrer Rolle als HelferInnen, welche sie aus dem (bei Ehrenamtlern subjektiv perzipierten) Vorsprung an Sprachkompetenz, Geld, Befugnissen, Eigentum, Wissen über Spielregeln etc. übernehmen.

Nach einem Überblick gängiger Integrationsvorstellungen und -konzepte legt der Beitrag einen Fokus auf raumbezogene Implikationen der „Integration“. So wird u.a. seit der Veröffentlichung des Race-Relation Cycle der Chicago School räumlich agglomerierten ethnischen Communities auf Quartiersebene ein besonderer Stellenwert für die Einführung von Neuzuwanderern in die Ankunftsgesellschaft zugesprochen. Auch neuere Untersuchungen (Ager und Strang, 2008; Aumüller und Bretl, 2008; Glick-Schiller und Caglar, 2011) unterstreichen den besonderen Einfluss lokaler Kontexte auf Integrationsprozesse von Zuwanderern auch über die Quartiersebene hinaus. Demnach wäre zu erwarten, dass Prozesse der Ankunft und des Einlebens in Großstädten unter anderen Bedingungen ablaufen, als in ländlichen bis mittelstädtischen Umgebungen. Vor diesem Hintergrund liegen der vorliegenden Studie folgende Fragestellungen zugrunde:

- Welche subjektiven Vorstellungen haben Menschen mit Fluchterfahrung über ein subjektiv zufriedenstellendes Einleben im Ankunftskontext? Welche Vorstellungen haben BetreuerInnen von Geflüchteten über ein gelungenes Einleben bzw. ,integriert sein“ der von ihnen Betreuten?

- Inwiefern bilden diese individuell-subjektiven Vorstellungen auch die in der öffentlichen Diskussion zirkulierenden Integrationskonzepte und -theorien ab?

- Treten in den subjektiven Vorstellungen über gelungenes Einleben auch raumbezogene Faktoren auf und wenn ja mit welcher Bedeutung? Werden diese Vorstellungen z.B. davon beeinflusst, dass ein Individuum in einem großstädtischen oder in einem klein/mittelstädtischen Umfeld angesiedelt ist?

Die nachfolgend präsentierten Ergebnisse sind Teil eines vom Ministerium für Innovation, Wissenschaft und Forschung des Landes Nordrhein-Westfalen über das Forschungsinstitut für gesellschaftliche Weiterentwicklung (FGW) geförderten Forschungsprojekts zu Integrationsprozessen asylberechtigter Flüchtlinge in nordrheinwestfälischen Städten und Gemeinden.

Im Folgenden werden zunächst allgemeine Integrationstheorien und -konzepte sowie deren Raumbezug zur Diskussion gestellt. Anschließend werden das methodische Vorgehen erläutert und die empirischen Ergebnisse präsentiert. Diese werden differenziert nach den subjektiven Integrati- onsvorstellungen der Geflüchteten und der in der Betreuung von Geflüchteten Tätigen. In einem Fazit werden beide Sichtweisen verglichen und in Relation zu einer postmigrantischen Perspektive auf Gesellschaft gebracht.

\section{Konzeptionelle Grundlagen: Eine kritische Betrachtung gängiger Integrationsmodelle und die Rolle des (Sozial-)Raums}

\subsection{Von der Assimilation zu gleichberechtigter Partizipation und Teilhabe?}

In der Wissenschaft konkurrieren verschiedene Integrationsmodelle (vgl. Überblick bei Fincke, 2008:21ff.): Das klassische Assimilations-Modell der Chicagoer Schule geht davon aus, dass sich Zuwanderer langfristig an ein etabliertes Gesellschaftssystem angleichen. Eine Grundannahme ist, dass Integrationsprozesse im Sinn eines einseitigen Anpassungsprozesses allein von der zuwandernden Bevölkerung ausgehen. Das Modell der partiellen bzw. ungleichmäßigen Assimilation geht einen Schritt weiter und differenziert darüber hinaus zwischen unterschiedlichen Bereichen (Beruf, Freunde, Kultur) sowie unterschiedlichem Tempo und Ausmaß der Anpassung an die Aufnahmegesellschaft, wobei eine letzte Stufe vollständiger Assimilation häufig ausbleibt. Es wird daher davon ausgegangen, dass es - unabhängig von der Aufenthaltsdauer - eher zu einem Nebeneinanderleben von $\mathrm{Zu}$ wanderern und Einheimischen mit allenfalls partieller Assimilation kommt (Treibel, 2008:109f, ähnliche auch in der deutschen Diskussion Esser, 2001 und Heitmeyer, 1998).

Neuere, in den USA entwickelte, Theorieansätze nehmen die differenzierten und variablen Bedingungen in der Aufnahmegesellschaft stärker in den Blick. So geht die Theorie der transnationalen Pluralisierung (Levitt und Waters, 2002) davon aus, dass bei begrenztem Arbeitsmarktzugang und einer großen Zahl von Zuwanderern gleicher Herkunft gleichzeitig Netzwerke zur Aufnahmegesellschaft sowie verstärkt zum Herkunftsland gepflegt werden. Die Nutzung der sozialen und ökonomischen Ressourcen eines transnationalen Raums begünstigt bikulturelle Kompetenzen, die im Sinn von Entwicklungspotentialen interpretiert werden. Die Theorie der segmentierten Assimilation (Portes, 1999; Portes und Rumbaut, 2006) thematisiert dagegen vor allem konflikthafte Konstellationen: Sie verfolgt die These, dass bei bestehendem Rassismus und schlechten Chancen des Arbeitsmarktzugangs eine Integration in endogene marginalisierte Gruppen erfolgt, deren Oppositionskultur gegen den mehrheitsgesellschaftlichen Mainstream übernommen wird. Die neoklassische Theorie (Alba und Nee, 2003) hingegen postuliert, dass sich die Zugewanderten bei funktionierenden Gesetzen gegen Diskriminierung, gleichen Bildungschancen und einem meritokratischen Aufstiegssystem so weit wie nötig an die Normen des sich ebenfalls verändernden Mainstreams anpassen, da sie nach Verbesserung ihrer Lebenschancen und sozialem Aufstieg streben. 
Die gesellschaftspolitische Diskussion in Deutschland wird seit den 1970er Jahren im starken Maß durch einen konservativen Integrationsbegriff geprägt. Dieser orientiert sich an der Vorstellung, es gäbe eine etablierte Kerngesellschaft, an die sich Menschen mit Migrationsbiographie einseitig anpassen müssten. Damit steht diese Auffassung tendenziell in einer Linie mit traditionellen Assimilationsmodellen (u.a. Esser, 2001; Heitmeyer, 1998). Traditionelle Integrationspolitik versucht entsprechend dieser Logik, Defizite bei den MigrantenInnen $\mathrm{zu}$ beseitigen. Integrationshindernisse werden vorzugsweise bei den kulturellen bzw. religiösen Andersartigkeiten oder in der Person des Migranten gesehen, nicht in den Bedingungen der Aufnahmegesellschaft. Vor dem Hintergrund zunehmender gesellschaftlicher Mobilität, umfassender globaler Wanderungsbewegungen und der wachsenden Bedeutung von Lebensformen die in mehreren lokalen und nationalen Kontexten verankert sind, stehen Vorstellungen eines homogenen, stabilen gesellschaftlichen Mainstreams jedoch immer weniger in Einklang mit der gesellschaftlichen Realität.

Das traditionelle Verständnis von Integration vor diesem Hintergrund als unzeitgemäß ablehnend, wird aus einer postmigrantischen Perspektive ein Paradigmenwechsel gefordert. Dieser zielt im Kern darauf ab, die Dichotomie von „Einheimischen“ und „MigrantInnen“ zugunsten einer BürgerIdentität mit gleichen Teilhaberechten und Partizipationschancen für alle aufzulösen. Gesellschaftliches Leitbild ist die Einheit der Verschiedenen, die „Integration“ jedem Bürger offen stellt (Foroutan, 2015:6). Das Konzept der postmigrantischen Gesellschaft hat Merkmale einer Forschungsperspektive und einer normativen Gesellschaftsvision zugleich. In einer ursprünglichen und wesentlichen Stoßrichtung geht es darum, die Einteilung der Gesellschaft in Gruppen nach Herkunft und „migrantisch“ versus ,einheimisch“ kritisch zu hinterfragen und letztendlich zu überwinden, da inzwischen die ganze Gesellschaft direkt oder indirekt durch Migration geprägt ist (u.a. Langhoff, 2011). Die Realität transnationaler und translokaler Verflechtungen anzuerkennen, zu analysieren und ihr gesellschaftspolitisch Rechnung zu tragen, ist ein zentrales Anliegen postmigrantischer Autoren (Tsianos und Karakayali, 2014; Foroutan, 2015; Römhild, 2015). Diese Kernidee wird mit unterschiedlichen Schwerpunkten angereichert. Während einige Autoren die Aufmerksamkeit auf die selbstintegrativen Leistungen verschiedener Generationen der so genannten Gastarbeiter-Zuwanderung lenken (z.B. Yildiz, 2015), rücken andere die Auflehnung gegen zunehmende gesellschaftliche Heterogenität und die daraus resultierenden Konflikte als typische Phänomene einer postmigrantischen Gesellschaft in den Fokus (Spielhaus, 2014). Die Auseinandersetzung mit gesellschaftlichen Aushandlungsprozessen um das Verständnis von „Integration“ aus postmigrantischer Perspektive, welche thematisiert, wie sich Differenzierungskriterien der Zuschreibung von Fremdheit verändern, unscharf werden oder sogar auflösen können, weist Parallelen zu einem sozialkonstruktivistischen Integra- tionsverständnis auf (Abramson, 1980:150; ,,boundary blurring“, vgl. Bauböck, 1995).

\subsection{Raumbezogene Implikationen - Integration und Segregation}

Aus raumsoziologischer bzw. sozialgeographischer Perspektive wird dem sozialräumlichen Kontext eine bedeutende Rolle beim Integrationsprozess von Zuwanderern beigemessen. Besonders in der deutschen Diskussion mit ihrem traditionellen Integrationsbegriff, welcher von Anpassung der MigrantInnen an einen gesellschaftlichen Mainstream ausgeht, werden Quartiere, die durch Konzentration von $\mathrm{Zu}$ gewanderten geprägt sind, tendenziell problematisiert. Diese defizitorientierte Perspektive auf Migration, die sich unter anderem im Etikett einer „Parallelgesellschaft“" wiederfindet (Yildiz, 2017:20), geht von einer langfristig negativen Wirkung ethnischer Segregation auf Integrationsprozesse aus. Zugleich wird die Gefahr einer zunehmenden Abkopplung von sich mit ihrem Raum identifizierenden ethnischen Communities vom Rest der Stadtgesellschaft gesehen, die mit wachsenden gesellschaftlichen Konflikten einhergehen kann (Teltemann et al., 2013:6). Laut Heitmeyer (1998) begünstigt eine ethnische Konzentration in infrastrukturell benachteiligten Gebieten Abhängigkeiten der MigrantInnen von ethnischen und religiösen Gruppierungen und gefährdet dadurch das Ein- und Zusammenleben. Für die Bewertung von Segregation als „Integration verhindernd“ ist die Kontakthypothese zentral: Diese besagt, dass durch - vor allem residenzielle - Nähe die Kontakthäufigkeit zwischen Individuen im Wohnumfeld steigt, mithin in einem ,gemischten“ Setting das Wissen übereinander und die Anpassung an die Verhaltensweisen des Mainstreams. Bei ethnisch homogenen Kontakten muss dieser Anpassungsprozess demnach ausbleiben (Häußermann und Siebel, 2001:45). Ähnlich problematisch wird ethnische residenzielle Segregation auch im Ansatz der segmentierten Assimilation interpretiert: Die räumliche Konzentration der Zuwanderer in Quartieren der endogenen marginalisierten Bevölkerung beschleunigt den sozialen Abstieg z.B. über schlechte Bildungsinfrastruktur, mangelnde Zugänge zu Informationen und Jobs sowie fehlende Rollenvorbilder (Portes, 1999).

Eine grundsätzlich positive Sicht auf migrantischethnische Segregation besitzt dagegen das Konzept der ethnischen Kolonie oder der ,arrival city“ (Saunders, 2011). Dieses geht davon aus, dass die Kolonie der Zugewanderten durch freiwillig aufgenommene und selbst organisierte Beziehungsstrukturen gekennzeichnet ist, die der Selbsthilfe in der Ankunftssituation dienen. Ethnische Kolonien oder auch „Ankunftsquartiere“ bieten Halt sowie Orientierung und stellen somit Brücken in die Aufnahmegesellschaft dar. Merkmale sind eine spezifische institutionelle Struktur mit religiösen Gemeinden, konfessionellen Schulen, politischen Zusammenschlüssen, Selbsthilfeorganisationen und Vereinen sowie eine migrantische Ökonomie (Tel- 
temann, 2013:7). Aus der Perspektive der Theorie transnationaler Pluralisierung können räumlich segregierte ethnische Gemeinschaften insbesondere über ihre Ökonomie die transnationale Existenz fördern. In eine ähnliche Richtung argumentiert Yildiz (2017:27f.), der aufzeigt, dass die Gastarbeiter in Deutschland z.B. entscheidend zur Wiederbelebung und Sanierung heruntergekommener urbaner Räume beigetragen haben. Ökonomische Selbstintegration als Überlebensstrategie unter marginalisierten gesellschaftlichen Bedingungen und somit ein gesellschaftlich nicht vorgesehenes „Ankommen auf eigene Rechnung" konnte mit einem sozialen Aufstieg der Zugewanderten durch Eigeninitiative einhergehen. Yildiz betrachtet diese Quartiere jedoch nicht als funktional für eine notwendige Anpassung der MigrantInnen an die Mehrheitsgesellschaft. Vielmehr geht es darum, die von den Newcomern selbst (mit-)gestalteten urbanen Räume als eine eigenständige Mischung von lokalen und globalen Bezügen als Resultat transkultureller urbaner Praktiken erkennbar zu machen. Auf diese Weise entstanden Transtopien, die sich aus Herkunfts- und Ankunftsräumen zu Alltagskontexten verdichten (Yildiz, 2017:24). Im Ansatz der neoklassischen Integrationstheorie ist sozialräumliche Segregation desgleichen nicht von funktionaler Bedeutung. Die postmigrantische Perspektive steht im Wiederspruch zu Konzepten, die darauf ausgerichtet sind, Wohnstandorte implizit oder explizit nach den Kriterien Migrationshintergrund bzw. Ethnizität zu differenzieren und zu bewerten. Vielmehr geht es darum entsprechende kausale Verknüpfungen im diskursiven Feld „Migration-Stadtraum-Problem“ zu dekonstruieren.

Alle klassischen Integrationstheorien arbeiten mit einer Dichotomie von Einheimischen und Zugewanderten. In einem postmigrantischen Verständnis jenseits dieser Dichotomie muss der Begriff „Integration“ ersetzt werden durch ein Zurechtkommen und heimisch werden von Individuen, unabhängig von kulturellen, nationalen oder ethnischen Zuschreibungen. Studien zur Ortsbindung in Deutschland haben gezeigt, dass bei gesichertem Einkommen mit der Wohndauer grundsätzlich und unabhängig von der Herkunft die sozialen Beziehungen, damit Vertrautheit und eine emotional positive Wahrnehmung des Wohnquartiers und der Gesamtstadt wachsen, sofern das eigene Wohnviertel nicht als marginalisierter, stigmatisierter Raum empfunden wird (Reuber, 1993; Sachs, 1993; Weiss, 1993; Köchling-Farahwaran, 2019). Ein dementsprechend interpretiertes Verständnis von „Integration" als ein "Sich-zurechtfinden“ in neuer Umgebung, liegt der vorliegenden Studie zugrunde.

\subsection{Integration - eine Frage des räumlichen Maßstabs?}

Relevante räumliche Kontexte werden innerhalb der Integrationstheorien - im Sinne der Kontakthypothese - vorwiegend lokal, auf der Quartiersebene identifiziert. Faktisch ist davon auszugehen, dass Kontexte auf verschiedenen Maßstabsebenen (scales) wirken. Scale wird hier in Anlehnung an Swyngedouw (1997) und Brenner (1997) als materielles Produkt sozialer Praktiken im Hinblick auf Governance, Einfluss, Identitäten etc. verstanden (vgl. auch Porst und Sakdapolrak, 2017). Manche für Integration bzw. das Einleben relevante Aspekte sind einer nationalen Ebene zuzuordnen (z.B. Landessprache, zahlreiche Gesetze und Konventionen; bewusste Wahl eines Landes als Ziel der Migration, nationale mental maps bzw. Stereotype; weiterhin bestehende transnationale Beziehungen zum Herkunftsland oder Zwischenstationen der Migration). Manche Aspekte unterscheiden sich auf Ebene des Bundeslandes (z.B. Verteilungsmodi für FluchtmigrantInnen) oder der Region (z.B. Dialekte, Traditionen) oder zwischen Regionstypen, wie städtische versus ländliche Räume (z.B. Idee der Stadt als „Integrationsmaschine“ über Vielfalt der Chancen). Das Einleben findet aber auch unterhalb der Quartiersebene im kleinräumigen Kontext des Wohngebäudes (z.B. Gruppenunterkunft, Zahl der Mietparteien) und der Wohnung (z.B. Ausstattung, Zustand) statt.

Inwiefern die verschiedenen Bezugsebenen der Integration im Hinblick auf die besondere Situation der Zuwanderung von FluchtmigrantInnen relevant sind, wurde bislang kaum diskutiert. Aumüller und Bretl (2008) sowie GlickSchiller und Çağlar (2011) gehen davon aus, dass Integrationsprozesse von MigrantInnen und Geflüchteten durch den lokalen Kontext auf Gemeinde- und Quartiersebene beeinflusst werden. Wichtige Variablen sind die Größe einer Stadt und die Zusammensetzung der Bevölkerung, die regionale Struktur des Arbeitsmarktes sowie der zivilgesellschaftliche und kommunalpolitische Umgang mit Newcomern (Aumüller et al., 2015:118). Große Städte gelten dabei aufgrund der Vielfalt von Chancen traditionell gegenüber kleineren Ortsgrößen als ,integrationsfähiger“ und sind aus diesem Grund häufiger das Ziel von Zuwanderung als ländlich geprägte Räume (Göschel, 2001:6). Dem ländlichen Raum wird demgegenüber eine engere soziale Kohäsion der EinwohnerInnen durch Verwandtschaft, Freundschaften und Vereine zugeschrieben, die zu einer Abschottung und Exklusion von Fremden tendiert (Weichhart et al., 2006; Blank, 2011).

Eine Besonderheit der Fluchtmigration ist aber unter anderem die staatliche Zuweisung eines Wohnsitzes, die es den MigrantInnen nicht erlaubt, frei gewählte Zielorte anzusteuern bzw. zugewiesene Gemeinden nach kurzer Zeit wieder zu verlassen (Wohnsitzauflage für drei Jahre nach Erhalt des Schutzstatus), so dass etliche ihren Wohnsitz nicht in eine großstädtische „Integrationsmaschine“ verlegen können, sondern in Klein- und Mittelstädten verbleiben müssen. Insofern ist Liebig (2015) zuzustimmen, der am postmigrantischen Konzept die Fokussierung empirischer Studien auf großstädtische Kontexte kritisiert, die nicht repräsentativ für die gesamte deutsche Gesellschaft sind. Interessant ist in diesem Zusammenhang daher ein Vergleich der Integrationsbedingungen von großstädtischen und klein- bzw. mittelstädtischen Strukturen. Dies gilt auch aufgrund widersprüchlicher Befunde: Der Vorstellung einer größeren Integrationsfähigkeit von Stadt widerspricht beispielsweise Petermann (2002), der in einem allgemeinen Stadt-Land-Vergleich ermittelte, 
dass die soziale Integration im Sinne persönlicher Netzwerke kaum durch den Wohnort, sondern vor allem durch Persönlichkeitsmerkmale der Individuen beeinflusst wird. Entsprechend wird in der vorliegenden Studie den subjektiven Integrationskonzepten nicht nur in einer Großstadt (Köln), sondern auch für einen klein- und mittelstädtisch geprägten Raum (Kreis Heinsberg) nachgegangen.

Davon abgesehen beziehen sich existierende Studien zu Integrationsvorstellungen in Deutschland häufig auf spezifische AkteurInnengruppen, wie Spitzenverbände des Sports (Soeffner und Zifonun, 2008), spezifische ethnische Communities (Nestvogel, 2014) oder Surveys für die deutsche Bevölkerung (Foroutan et al., 2014; Zick und Preuß, 2016). Dabei wurden sowohl Personen, die in der Betreuung von Geflüchteten tätig sind als auch multiskalare, sozialräumliche Implikationen der Integration bislang kaum beachtet.

Im Mittelpunkt der folgenden Ausführungen stehen - im Unterschied zu den nur kurz angerissenen Theorien bzw. Modellen und Debatten - konkrete und individuelle Integrationsvorstellungen der Geflüchteten selbst und von Personen, die beruflich oder ehrenamtlich in der Hilfe für Geflüchtete tätig sind. Als auf „Integration“ bezogen wurden alle Äußerungen der InterviewpartnerInnen identifiziert, die sich auf ein Leben im Ankunftskontext beziehen. AkteurInnen der Betreuung von Geflüchteten wurden zudem gezielt und offen (ohne Vorgabe einer Definition) nach ihrem Integrationsverständnis gefragt; für die Geflüchteten wurde der Begriff mit „sich zuhause fühlen“ umschrieben. Entsprechend wird hier keines der beschriebenen Integrationskonzepte vorausgesetzt, favorisiert oder ein eigenes vertreten. Angestrebt ist eine größtmögliche Offenheit gegenüber den Vorstellungen der GesprächspartnerInnen im Zusammenhang mit Einleben, Zurechtkommen und Wohlfühlen im Ankunftskontext in systemischer, politischer und sozialer Hinsicht, funktional und emotional. $\mathrm{Zu}$ diesen Vorstellungen gehören wahrgenommene Komponenten und Mechanismen, sowohl Chancen als auch Barrieren. Diese Offenheit schließt Bezüge zu verschiedenen Maßstabsebenen einschließlich transnationaler Beziehungen ein.

\section{Methodik: Leitfadengestützte Tiefeninterviews}

Im Rahmen der vorliegenden Studie wurden mit insgesamt 41 Geflüchteten und 25 beruflich oder ehrenamtlich in der Flüchtlingsbetreuung tätigen Personen themenzentrierte Leitfadeninterviews durchgeführt. Die Interviews wurden in der Stadt Köln sowie in einigen Gemeinden des Kreises Heinsberg, gelegen zwischen Aachen und Düsseldorf an der Grenze zu den Niederlanden, durchgeführt. Hintergrund für die Wahl dieser Fallstudienorte war das Anliegen, mögliche Unterschiede hinsichtlich subjektiver Integrationsvorstellungen zwischen einem großstädtischen auf der einen und einem mittel- bis kleinstädtischen Kontext auf der anderen Seite identifizieren zu können. Köln ist als größte Stadt im Bun- desland Nordrhein-Westfalen sowie als viertgrößte Stadt in Deutschland ein wichtiges Oberzentrum mit einer Vielzahl von Einrichtungen der sozialen Hilfe und einem differenzierten Arbeitsmarkt. Der Kreis Heinsberg entspricht hingegen einer für das Bundesland typischen Region, die sich aus Klein- und Mittelstädten ohne ein dominierendes Zentrum zusammensetzt. Von den 1,08 Millionen Einwohnern der Stadt Köln im Jahr 2017 haben 19,3 \% eine ausländische Staatsbürgerschaft, bzw. insgesamt $38 \%$ einen Migrationshintergrund. Die Hauptherkunftsländer von FluchtmigrantInnen (Syrien, Iran und Irak) haben einen Anteil von 6,8\% an der ausländischen Bevölkerung Kölns. Von den KölnerInnen mit deutscher Staatsbürgerschaft besitzen weitere 18,5\% einen Migrationshintergrund. Die Stadt Köln beherbergt Ende 2017 ca. 10.200 Geflüchtete (Stadt Köln 2017). In den zehn Kommunen des Kreises Heinsberg leben etwa 260.000 EinwohnerInnen, von denen 14,7\% keine deutsche Staatsbürgerschaft besitzen, wobei dieser Anteil auf Grund historischer Entwicklungen in den einzelnen Gemeinden zwischen $8,4 \%$ und $38,8 \%$ stark schwankt. So ist hier zum Teil die Zuwanderung von Personen aus den angrenzenden Niederlanden von Bedeutung, zum Teil auch von MigrantInnen aus der „Gastarbeiter“-Zuwanderung in Zechen des Steinkohlebergbaus. Ende 2017 leben ca. 3.600 zugewiesene Geflüchtete im Kreisgebiet (Kreis Heinsberg 2018).

Bei den Fallbeispielen Köln und Kreis Heinsberg handelt es sich somit um Regionen, die von substanzieller Gastarbeiterzuwanderung sowie entsprechenden Erfahrungen im Umgang mit Zuwandernden gekennzeichnet sind. Sie unterscheiden sich diesbezüglich von ausgeprägt ländlich-peripheren Räumen oder Regionen Ostdeutschlands mit deutlich anderer Zuwanderungsgeschichte (z.B. Münch, 2013).

\subsection{GesprächspartnerInnen mit Fluchterfahrung}

Bei den 41 befragten Geflüchteten handelt es sich vorwiegend um Personen mit syrischer Staatsangehörigkeit, die auch den größten Anteil an den nach Deutschland einreisenden FluchtmigrantInnen repräsentieren (BAMF, 2017, Tabelle 1). Gemäß der Fragestellung des Gesamtprojekts, in der es darum geht, Prozesse des Ankommens in Deutschland auf Barrieren und Chancen hin zu untersuchen, wurden vorwiegend Personen mit Asylberechtigung (für 3 Jahre) oder subsidiärem Schutz (für $1 \mathrm{Jahr}$ ) befragt, aber auch Personen mit bereits längerem Aufenthalt in Deutschland, deren rechtlicher Status noch nicht geklärt war. Der $\mathrm{Zu}-$ gang zu dieser Personengruppe wurde über Organisationen oder Projekte der ehrenamtlichen Hilfe für Geflüchtete hergestellt (z.B. Deutsches Rotes Kreuz, Diakonie, Caritas, Integrationshaus, Kulturprojekte), wobei die Ehrenamtler geeignete Personen für Interviews vermittelten. Mithin handelt es sich um eine willkürliche Stichprobe, welche aus zweierlei Gründen nicht alle Gruppen von FluchtmigrantInnen abbildet: Zum einen müssen die Geflüchteten von sich aus 
Tabelle 1. Merkmale der GesprächspartnerInnen im Hinblick auf subjektive Vorstellungen zu Einleben/Integration von Geflüchteten in die Aufnahmegesellschaft.

\begin{tabular}{|c|c|c|}
\hline & Köln & Kreis Heinsberg \\
\hline \multirow[t]{6}{*}{ Gespräche mit Geflüchteten } & 9 Frauen, 12 Männer & 9 Frauen, 11 Männer \\
\hline & $\begin{array}{l}\text { Alter: unter } 20 \text { J. (4), 20-30 J. (8), } \\
\text { 31-40 J. (3), 41-50 J. (3), über } 50 \text { J. (3) }\end{array}$ & $\begin{array}{l}\text { Alter: unter } 20 \text { J. (3), 20-30 J. (8), } \\
\text { 31-40 J. (8), 41-50 J. (0), über } 50 \text { J. (1) }\end{array}$ \\
\hline & $\begin{array}{l}\text { Herkunftsland: Syrien (13), Irak (4), Iran (2), } \\
\text { Eritrea (1), Palästina (1) }\end{array}$ & $\begin{array}{l}\text { Herkunftsland: Syrien (8), Irak (4), Iran (3), Af- } \\
\text { ghanistan (3), Usbekistan (1), Tadschikistan (1) }\end{array}$ \\
\hline & $\begin{array}{l}\text { Dauer des Aufenthalts in Deutschland: ca. } \\
1 \text { Jahr (8), } 2 \text { Jahre (9), } 3 \text { Jahre (2), } 5 \text { Jahre (2) }\end{array}$ & $\begin{array}{l}\text { Dauer des Aufenthalts in Deutschland: ca. } 1 \\
\text { Jahr (1), } 2 \text { Jahre (12), } 3 \text { Jahre (6), } 5 \text { Jahre (1) }\end{array}$ \\
\hline & $\begin{array}{l}\text { Aufenthaltsrechtlicher Status: asylberechtigt } \\
\text { (10), subsidiärer Schutz (6), noch nicht ent- } \\
\text { schieden oder unklar (5) }\end{array}$ & $\begin{array}{l}\text { Aufenthaltsrechtlicher Status: asylberechtigt } \\
\text { (7), subsidiärer Schutz (5), noch nicht entschie- } \\
\text { den oder unklar (8) }\end{array}$ \\
\hline & $\begin{array}{l}\text { Eigene Wohnung (10), Zimmer in Heim/WG } \\
\text { (7), Zimmer mit Anderen (4) }\end{array}$ & $\begin{array}{l}\text { Eigene Wohnung (14), Zimmer in Heim/WG } \\
\text { (5), Zimmer mit Anderen (1) }\end{array}$ \\
\hline \multirow[t]{3}{*}{$\begin{array}{l}\text { Gespräche mit Personen, } \\
\text { die in der Betreuung von } \\
\text { Geflüchteten tätig sind }\end{array}$} & $\begin{array}{l}\text { beruflich-VertreterInnen von: } \\
\text { Stadtverwaltung } \\
\text { Jobcenter } \\
\text { Kommunales Integrationszentrum } \\
\text { Volkshochschule } \\
\text { Industrie- und Handelskammer } \\
\text { Städtische Wohnungsgesellschaft }\end{array}$ & $\begin{array}{l}\text { beruflich - VertreterInnen von: } \\
\text { Verwaltung der kreisangehörigen } \\
\text { Städte/Gemeinden } \\
\text { Jobcenter } \\
\text { Kommunales Integrationszentrum } \\
\text { Volkshochschule }\end{array}$ \\
\hline & $\begin{array}{l}\text { ehrenamtlich - VertreterInnen von: } \\
\text { Flüchtlingsrat } \\
\text { Caritas } \\
\text { In Via } \\
\text { Bürgerinitiativen der Hilfe für } \\
\text { Geflüchtete }\end{array}$ & $\begin{array}{l}\text { ehrenamtlich-VertreterInnen von: } \\
\text { Caritas } \\
\text { Bistum } \\
\text { Bürgerinitiativen der Hilfe für } \\
\text { Geflüchtete }\end{array}$ \\
\hline & 8 Frauen, 6 Männer & 6 Frauen, 5 Männer \\
\hline
\end{tabular}

Kontakt zu den Ehrenamtlern hergestellt oder aufrechterhalten haben, zum anderen handelt es sich um Personen mit einer gewissen Offenheit und Auskunftsbereitschaft gegenüber den Forschenden. Die Wahl der Sprache des Interviews war den GesprächspartnerInnen überlassen: Interviews auf Deutsch oder Englisch wurden von den Forschenden ohne, Interviews in Arabisch oder Farsi mit Hilfe von DolmetscherInnen durchgeführt. Bei den DolmetscherInnen handelte es sich um studentische Hilfskräfte des Forschungsprojekts oder selten um andere anwesende Geflüchtete. Auf die Probleme bei Interviews mit Fremdsprachlern, die erst einer Übersetzung bedürfen, wird von verschiedenen AutorInnen hingewiesen (z.B. Temple and Young, 2004; Filep, 2009; Inhetveen, 2012). Die Verschiebung von Bedeutungen durch Übersetzung, aber auch durch noch unvollständig ausgebildete Sprachkompetenz bei Verwendung des Deutschen kann durch Vergleich von Aussagen zu ähnlichen Themen ansatz- weise kontrolliert, aber nicht völlig ausgeschlossen werden. Die Gespräche wurden in der Regel in den Räumlichkeiten der Hilfseinrichtung geführt, sie dauerten zwischen $10 \mathrm{Mi}-$ nuten und anderthalb Stunden.

Der Interviewleitfaden umfasste folgende Themen: Die „Vorgeschichte“ der GesprächspartnerInnen im Herkunftsland bzw. auf der Flucht, Erfahrungen bei der Ankunft in Deutschland, Bewältigung des Alltags mit den relevanten Bereichen Sprache, Wohnung und Arbeit, relevante HelferInnen und wichtige Kontakte. Im Hinblick auf den Aspekt „Integration“ wurden die Geflüchteten nach dem aktuellen „Zuhause“ und den persönlichen Zielen für die nächsten 5 bis 10 Jahre gefragt. Weiterhin bedeutsam war in diesem Zusammenhang die Frage nach den Problemen, denen man sich im Ankunftskontext gegenüber sah. Begriffe wie „Integration“ und „Heimat“" wurden hier bewusst vermieden, um verschie- 
dene Interpretationen und mit den Begriffen möglicherweise assoziierte politische Konnotationen zu umgehen.

\subsection{In der Geflüchtetenhilfe tätige Gesprächspartnerlnnen}

Bei dieser Gruppe handelt es sich um Personen, die Geflüchteten beruflich oder ehrenamtlich Hilfe beim Ankommen in Deutschland anbieten. Mit VertreterInnen der Stadtverwaltungen, der kommunalen Integrationszentren und Integration Points, des Jobcenters, der Volkshochschule und der Industrie- und Handelskammer ist ein Teil der GesprächspartnerInnen dem Bereich der kommunalen Verwaltung zuzurechnen. Aus dem zivilgesellschaftlichen Bereich wurden Gespräche mit VerteterInnen der Caritas, der In Via, von Bürgerinitiativen und vom Kölner Flüchtlingsrat geführt. Ziel der Auswahl war es, mit Personen zu sprechen, welche die Situation der Geflüchteten aus eigener, primärer Erfahrung, auch im Detail und aus persönlicher Interaktion kennen; zudem sollten sie verschiedene Bereiche des Umgangs mit Geflüchteten abdecken (ehrenamtlich, administrativ, verschiedene Ämter und Organisationen). Die InterviewpartnerInnen wurden anhand von Berichten in lokalen Zeitungen, Homepages der Städte und Gemeinden sowie aus Empfehlungen von InterviewpartnerInnen identifiziert. Da insbesondere die ehrenamtlichen, aber prinzipiell auch die behördlichen HelferInnen, ihre Aufgabe freiwillig übernommen haben, ist schon vorab davon auszugehen, dass sie dem Thema Integration grundsätzlich positiv gegenüberstehen. In der Sichtweise und Bewertung dessen, was Integration ausmacht, sind aber dennoch Unterschiede möglich. Die Interviews wurden in den Büros der jeweiligen AkteurInnen geführt; sie dauerten zwischen 30 und 90 Minuten. Der Interviewleitfaden umfasste die Themen Sprachlernangebote und deren Nutzung, Zugänge der Geflüchteten zum Wohnungs- und Arbeitsmarkt sowie Einschätzung der sozialen Netzwerkeinbindung und der Integrationsprozesse im Allgemeinen mit Schwerpunkt auf der jeweiligen Expertise des Gegenübers. Im Zusammenhang mit den beobachteten Integrationsprozessen wurde auch gezielt nach dem Integrationsverständnis gefragt.

Alle Interviews wurden mit einem Diktiergerät aufgezeichnet und anschließend transkribiert. Die Auswertung erfolgte zunächst in der Logik einer qualitativen Inhaltsanalyse (Mayring, 2015). Ein deduktives Kategorienschema entlang der Leitfragen wurde induktiv ergänzt durch Aspekte, die von den GesprächspartnerInnen selbst ins Spiel gebracht oder betont wurden. Die Aggregierung der Kriterien wiederum ist der Logik einer Grounded Theory verpflichtet (Glaser und Strauss, 1979). Angestrebt wird dabei eine gegenstandsbezogene Theorie zum Thema „Integration“. Von diesem konzeptionellen Kernbegriff ausgehend, der in den Interviews mit „,sich zuhause fühlen“, „,sich einleben“, ,ankommen“ oder „persönliche Zukunft" umschrieben wurde, wurden Elemente gruppiert und nach ihrer Relation zum Kerngegenstand etikettiert, z.B. als grundlegende Voraussetzun- gen, nötige Anpassungen oder Barrieren der „Integration“. Dieses „,axiales Codieren“, im Sinn von ursächlichen Bedingungen und Kontexten sowie intervenierende Bedingungen, Handlungsstrategien und Konsequenzen, folgt im Grundsatz den Elementen des Codierparadigmas für ein zentrales Phänomen nach Strauss (Kuckartz, 2010:81). Entsprechend der Grundannahmen der Grounded Theory wird nicht nach individuellen Ansichten oder typischen Vorstellungsmustern differenziert. Vielmehr ist es Ziel alle Aspekte einzubeziehen, die seitens der Befragten mit dem Thema verbunden werden. Die dementsprechend aus dem empirischen Material abgeleiteten subjektiven Integrationstheorien der Geflüchteten sowie der AkteurInnen der Hilfe für Geflüchtete werden im Ergebnisteil entwickelt, anschließend im Fazit einander gegenübergestellt. Es werden jedoch keine Vergleiche individueller Argumentationsstrukturen untereinander oder im Hinblick auf sozialstrukturelle Merkmale der GesprächspartnerInnen (Alter, Geschlecht, Wohndauer, Familienstand, etc.) vorgenommen.

Die Aussagekraft der Ergebnisse muss dahingehend eingeschränkt werden, dass aufgrund des Zugangs nur Geflüchtete erfasst wurden, welche über Kontakt zu Ehrenamtlern verfügen, also in einem gewissen Maß freiwillig mit VertreterInnen der Aufnahmegesellschaft interagieren. Gleiches gilt umgekehrt für die in der Betreuung Tätigen. Damit sind Personen ohne oder mit nur oberflächlichem Kontakt zur jeweils anderen Personengruppe nicht abgebildet.

\section{Ergebnisse: Subjektive Integrationsvorstellungen von Geflüchteten und in der Flüchtlingsbetreuung tätigen Personen}

\subsection{Subjektive Integrationsvorstellungen von Geflüchteten}

Bei denjenigen Geflüchteten, die sich explizit zu ihrem Zuhause-Fühlen am Aufenthaltsort geäußert haben, reicht die Spannweite von eindeutig positiven Bezügen (z.B. „Deutschland ist wie meine Heimat, mein zweites Land" (Herr T, 19 Jahre, Köln, aus dem Irak)) bis zu Ausdruckformen einer deutlichen Ablehnung, wie z.B. „Wenn ich meine Uni fertig mache, mein Zertifikat habe, dann fliege ich irgendwo hin, irgendwo hin, Australien oder Kanada oder irgendwo hin. Das ist vielleicht nicht besser, aber besser als hier" (Herr A, 23 Jahre, Köln, aus Syrien). Dennoch lassen sich aus den Interviews gemeinsame Grundelemente einer subjektiven Theorie des Zuhause-Seins in Deutschland herausarbeiten, in der sich typische Faktoren - fördernde und hinderliche - identifizieren lassen.

\section{a) Kernelemente des Zuhause-Fühlens}

Bei der Frage nach den Bedingungen dafür, sich in Deutschland zuhause zu fühlen bzw. hier seine Zukunft zu sehen, kommt der Präsenz der eigenen Kernfamilie (PartnerIn und 
Kinder) eine zentrale Bedeutung zu: „Without family reunion no integration" (Herr A, 38 Jahre, Heinsberg, aus Syrien). Sorge um nahe stehende Familienangehörige blockieren weitgehend andere Bestrebungen, sich in der Aufnahmegesellschaft einzuleben, wie z.B. die Bemühungen im Bereich des Spracherwerbs. Die eigenen Eltern sind dem gegenüber grundsätzlich weniger relevant, besitzen jedoch für junge Alleinreisende eine hohe Bedeutung: So bekennt ein 18-jähiger Mann aus Syrien: „Deutschland ist wie meine Heimat, mein zweites Land. Wenn meine Mutter hier ist, dann ganz Heimat" (Herr F, 18 Jahre, Köln, aus Syrien), da hier Eltern oder Elternteile eine große Bedeutung für den emotionalen Rückhalt besitzen, als Vertrauenspersonen, mit denen Alltagssorgen besprochen werden können. Auch die zukünftige Familie, die man in Deutschland erst noch gründen möchte oder ein hier zu findender Partner kann Perspektiven auf eine künftige Verankerung in der Ankunftsgesellschaft eröffnen.

Als weiteres zentrales Element eines Sich-ZuhauseFühlens wird die Sicherheit hervorgehoben. Die Konnotationen von Sicherheit gehen in verschiedene Richtungen: Sicherheit vor den Auswirkungen eines (Bürger-)Kriegs (Bombardierungen, Hunger, weder Wasser noch Elektrizität), Sicherheit vor Übergriffen anderer, wie sie z.B. von Mitgliedern religiöser oder ethnischer Minderheiten oder auf der Flucht erlebt wurden (Beschränkungen, Bedrohung, Vergewaltigungen), aber auch Rechtssicherheit (keine Korruption, transparente Regeln und Ordnung). Diese Rechtssicherheit wird nicht nur als Verlässlichkeit ohne Bestechung oder Vetternwirtschaft aufgefasst, sondern auch als Chance, seine Ziele realisieren, seine Träume verwirklichen, seinen Weg aus eigener Kraft gehen zu können, ohne dass die soziale Umgebung oder das Gesellschaftssystem diesen Weg behindern ,So for me, what makes me say that I am here in my country, is that I can do anything I dream about, there is no obstacles" (Frau E, 21 Jahre, Köln, aus Syrien). Sicherheit bietet auf der Makroebene der gesellschaftliche Rahmen in Deutschland, auf der Mikroebene ist es die eigene Wohnung, welche Privatsphäre und Ruhe gewährt, ein Refugium in Kontrast zu der kollektiven Enge von Flucht und Unterbringung in Gemeinschaftsunterkünften.

Als drittes zentrales Element kann der Bereich Arbeit und Einkommen identifiziert werden. Arbeit wird dabei mehrheitlich nicht als Job verstanden, sondern als Verwertung einer Qualifikation im Anschluss an eine berufliche oder hochschulische Ausbildung. Betont wird die Bedeutung von Arbeitsstelle und Einkommen für die eigene Unabhängigkeit: „When I go to work I think it will be more better. You are self confident, you own your own money, you can do what you want" (Frau T, 36 Jahre, Heinsberg, aus Usbekistan). Einkommen aus eigener Arbeit wird als Grundlage für ein „,normales“ Leben und damit als zentral für das Wohlbefinden in der Ankunftsgesellschaft betrachtet. Unabhängig sein, über sich selbst bestimmen können wird auch als Schwelle genannt, jenseits derer man sich nicht mehr als „Flüchtling" fühlt. Eltern schließen dabei die Zukunft ihrer Kinder in Form geregelter Schul- und Berufsausbildung als Basis für spätere Erwerbstätigkeit mit ein.

Das Beherrschen der deutschen Sprache wird sowohl als ein zentrales Element des Sich-Zuhause-Fühlens als auch als eine notwendig empfundene Voraussetzung dafür bewertet: „Sprache ist der Schlüssel, der Türen öffnet" bringt es einer der Befragten auf den Punkt (Herr S, 21 Jahre, Köln, aus Syrien). Die Gespräche machten immer wieder große Bemühungen um Zugänge zur deutschen Sprache deutlich, da Sprache vor allem für das Ziel von Ausbildung, Studium und Arbeitsaufnahme als unabdingbar begriffen wird. Spracherwerb verbessert aber auch die Selbständigkeit im Alltag und die erlebte Akzeptanz durch Mitglieder der Aufnahmegesellschaft. Wer noch über geringe Sprachkompetenzen verfügt, berichtet über wahrgenommene Distanzen bei Behörden und im Alltag. Die InterviewpartnerInnen streben daher engagiert nach Aufnahme in Sprachkurse; tendenziell extrovertiertere oder fremdsprachen-affine Personen betreiben den Erwerb der deutschen Sprache auf eigene Faust (z.B. Nutzen von Online-Kursen zur Schulung, Kontakt zu deutschsprachigen Personen für Sprachpraxis).

Trotz gefühlter Bindungen wurde eine Rückkehr in das Herkunftsland von den GesprächspartnerInnen implizit oder explizit weitgehend ausgeschlossen. Manche bekunden eine auf emotionaler Verbindung beruhende moralische Verpflichtung, dem Herkunftsland zu helfen, sich zum Beispiel in Syrien am Wiederaufbau nach dem Bürgerkrieg zu beteiligen. Wird der Gedanke an eine Rückkehr aber explizit reflektiert, so wird er letztlich verworfen. Gründe hierfür sind die vermutete lange Dauer des Bürgerkriegs, das Andauern politischer Repression selbst nach einem Kriegsende oder die mit der Zeit wachsende Einbindung in die Aufnahmegesellschaft. „Mein Land braucht mich zum Wiederaufbau, dann ist aber alles verloren, was ich hier gemacht habe" (Herr D, 23 Jahre, Köln, aus Syrien). Ein neu aufgebautes Leben, die erworbene Sprach- und Systemkenntnis, die gewonnene Arbeitsstelle und die für den Alltag relevanten Sozialkontakte werden wahrscheinlich nicht mehr aufgegeben, mit Sicherheit nicht, wenn erst die eigenen Kinder in Sprache und Spielregeln der Ankunftsgesellschaft eingewöhnt sind. Des Weiteren ist bei vielen InterviewpartnerInnen die soziale und materielle Basis im Herkunftsland längst erodiert: das Haus der Familie zerstört, deren Mitglieder auf verschiedene Orte und Länder verteilt. Für Personen, die aus politischen, religiösen oder ethnischen Gründen geflohen sind, stellt sich die Frage einer Rückkehr ohnehin nicht mehr, da diese radikale gesellschaftlich-politische Reformen voraussetzen würde.

\section{b) Das Erleben von Fremdheit und das Verhältnis zur Herkunftsgesellschaft}

Von den GesprächspartnerInnen wird mehr oder minder explizit ein funktionales Sich-Zuhause-Fühlen von einem emotionalen Bezug unterschieden. Manche betonen hier, dass sie sich kulturell bzw. vom Lebensgefühl her nicht in einem 
Land heimisch fühlen können, in dem sie nicht aufgewachsen sind. Als intervenierender Faktor, welcher das Fremdheitserleben in der Aufnahmegesellschaft beeinflusst, wurde aus den Gesprächen heraus das individuelle Erleben von Fremdheit im Herkunftsland identifiziert. Geflüchtete, die in ihrem Herkunftsland sozial ungünstigen Lebensbedingungen in ihrer Familie und im weiteren Umfeld ausgesetzt waren, wie Angehörige religiöser oder ethnischer Minderheiten, brachten oft weniger zum Ausdruck, eine emotional bindende Heimat verloren zu haben. Eine Sunnitin, welche andauernde familiäre Streitigkeiten und schließlich die Trennung ihrer Eltern wegen religiöser Differenzen miterleben musste, nimmt die religionstolerante Situation in Deutschland emotional positiv an: „I can live in peace without religious sectarianism problems and I raise my child far away from those problems and racism in a way" (Frau E, 21 Jahre, Köln, aus Syrien). Ähnlich äußern sich Kurden, die in der Schule ihre Muttersprache nicht benutzen durften oder Christen, die öffentlich kein Kreuz tragen konnten oder sich zum Tragen eines Kopftuchs genötigt sahen. In anderen Gesprächen war das Herkunftsland auch emotional positiv konnotiert, indem beispielsweise ein harmonisches soziales Umfeld ohne Diskriminierungen thematisiert wurde. Der Fluchtgrund lag hier auch nicht in Repressalien des Alltags begründet, sondern beispielsweise in unmittelbaren Einwirkungen des Bürgerkriegs (Zerstörung oder Okkupation des Wohnortes durch eine andere Armee) oder der drohenden Einberufung zum Militärdienst. Wird der Verlust eines spezifischen, als positiv empfundenen Lebensgefühls - häufig ausgedrückt in Begriffen wie Freundeskreis, Gelassenheit, familiärer Zusammenhalt, Gemeinschaft - beklagt, kann es schwer erscheinen, sich in Deutschland emotional einzuleben. Zusammenkünfte mit Freunden bei landestypischen Mahlzeiten und Reden über alte Zeiten können hier - vergleichbar einer nostalgischen Reaktion (Weiss, 1993:81f.) - eine temporäre emotionale Rückkehr in die alte Heimat ermöglichen.

\section{c) Wahrgenommene Brücken zur Aufnahmegesellschaft}

Als Grundlage dafür, in der neuen Gesellschaft subjektiv eine Zukunft wahrnehmen zu können, und um in einen $\mathrm{Zu}$ stand des Sich-Zuhause-Fühlens zu gelangen, muss ein Mindeststatus des gefühlten Aufgenommenseins erreicht werden. Wie in den Gesprächen deutlich wurde, gehören dazu zum einen konkrete rechtliche Bedingungen, wie die behördliche Aufenthaltsberechtigung (Asylstatus oder subsidiärer Schutz), zum anderen das Gefühl, von der Aufnahmegesellschaft grundsätzlich akzeptiert zu werden. Für das Gefühl von Akzeptanz sind Kontakte jenseits von Kontakten zwischen Geflüchteten innerhalb von Unterkünften, Integrations-/Sprachkursen oder Behördenfluren, von hoher Relevanz: Dazu zählen die erlebte Freundlichkeit und Hilfsbereitschaft sowohl im Alltag als auch bei Behörden und die konzentrierte Hilfsbereitschaft ehrenamtlicher Initiativen. Bedingt durch die Stichprobengenerierung (vgl. Kap. 3) besaßen alle GesprächspartnerInnen Kontakt zu ehrenamtlichen Initiativen bzw. Projekten. MitarbeiterInnen dieser Initiativen nahmen die Rolle von Vertrauenspersonen ein, die im Alltag helfen und in einigen Fällen zu den wichtigsten AnsprechpartnerInnen bei Problemen wurden. Die Schwierigkeit, Kontakt zu Menschen in der Aufnahmegesellschaft zu knüpfen, wird unterschiedlich eingeschätzt; Leute kennen zu lernen kann als schwer empfunden zu werden, aber auch als leicht „Wenn man nett ist, kann man viele Leute kennen lernen“ (Herr K, 18 Jahre, Köln, aus Syrien). Abhängig von individueller Kontaktfreudigkeit wurden diverse Strategien genutzt, um mit Einheimischen in Kontakt zu kommen, z.B. Fußball spielen im Verein oder Besuch eines Fitnessstudios. „It's good if someone has a hobby in Germany, then people help him to do better" (Herr F, 18 Jahre, Köln, aus Syrien). Das aktive Engagement in der ehrenamtlichen Initiative als HelferIn für andere Geflüchtete (z.B. bei Übersetzungen, Begleitung auf Ämter) macht die Fluchtbetroffenen nicht nur zu EmpfängerInnen von Hilfe, sondern zu gleichwertigen PartnerInnen ihrer ehrenamtlichen KollegInnen (zu Geflüchteten als HelferInnen siehe auch Adam et al., 2019).

\section{d) Wahrgenommene Barrieren der Aufnahmegesellschaft}

Ein Sich-Zuhause-Fühlen in Deutschland kann durch negative Erlebnisse mit VertreterInnen der Ankunftsgesellschaft behindert oder sogar deutlich gestört werden. Eine zentrale Barriere ist erlebte Diskriminierung. Die GesprächspartnerInnen mit Fluchterfahrungen unterscheiden hier sehr deutlich zwischen beispielsweise einerseits bürokratischen Verfahren, welches als Tribut an die Ordnung und Rechtssicherheit des Landes akzeptiert werden und andererseits wahrgenommener und erlebter Ungleichbehandlung und Ungerechtigkeit. Berichtete negative Erlebnisse konzentrieren sich auf das Aufsichtspersonal in Flüchtlingslagern (z.B. rüder Tonfall, Behandlung von oben herab), manche VertreterInnen von Behörden (unangemessenes Beharren auf fließendem Beherrschen der deutschen Sprache oder auf Pünktlichkeit) sowie manche Vertreter der Polizei (Razzien/Repressalien für alle bei Fehlverhalten einzelner, schärfere Kontrollen allein aufgrund eines fremdländischen Aussehens, pauschaler Kriminalitätsverdacht, keine Entschuldigung bei Missverständnissen). Das Erleben von Diskriminierung kann auch subtiler sein, etwa wenn sich in der Straßenbahn andere Personen nicht neben jemanden setzen oder unpersönlich aufgrund von Berichterstattung in den Medien. Öffentliche Pauschalisierungen im Umgang mit dem Thema Migration nach den Übergriffen mehrheitlich nordafrikanischer Zuwanderer auf in der Domumgebung feiernde Frauen in der „,Kölner Silvesternacht“ 2016/17 (vgl. Guinan-Bank, 2017) hat gerade bei jungen arabischen Männern eine Verunsicherung im Alltag ausgelöst. Das Gefühl, stigmatisiert und unerwünscht zu sein, hat die unbefangene Kontaktaufnahme in Alltagssituationen erschwert. Der Blick auf die parallele politische Diskussion lässt den Eindruck entstehen, dass Geflüchtete als 
„Ware“ oder Manövriermasse der politischen Stimmung geopfert werden, indem man nach Belieben Bedingungen verschärft. Diskriminierung wird weiterhin durch Ausnutzen besonders auf der Arbeitsstelle erlebt. Aus Unkenntnis über die eigenen Rechte werden Geflüchteten unangemessene Tätigkeiten, Arbeitzeiten oder untertarifliche Bezahlungen zugemutet. Auch der formelle Wohnungsmarkt über Meldungen auf Inserate in Zeitungen oder Internet wird als tendenziell diskriminierend erlebt: Bei Anfragen erhalten Geflüchtete Absagen aufgrund ihres fremd klingenden Namens, weil sie „Flüchtlinge“ sind, oder weil Vermieter die Miete nicht vom Jobcenter bzw. Sozialamt bezahlt haben möchten. So kann eine erfolgreiche Vermittlung oft nur durch direkte $\mathrm{Zu}$ weisungen von Behörden oder auf einem informellen Markt über Bekannte oder Ehrenamtler erzielt werden. Gelegentlich wird auch über Sozialneid berichtet, u.a. wenn Geflüchteten das Gefühl vermittelt wird, keine Ansprüche stellen zu dürfen, wie z.B. den Wunsch nach einem eigenen Handy oder Auto, da ein „Flüchtling“ in Armut zu leben habe. „For example you have worked and afforded to buy a car. That did not happen to me, but with my brother. [...] He worked and afterwards he bought a car, then [they make him feel] like he does not need to do it, as if he does not need it, something extra. Here it feels, some racism, as they don't want us to live in luxury" (Frau Q, 50 Jahre, Köln, aus Syrien).

Die Bevölkerung der Aufnahmegesellschaft wird am ehesten nach freundlichen und unfreundlichen Menschen differenziert: „Die Leute sind manchmal schlecht und manchmal gut, wie überall " (Herr I, 18 Jahre, Köln, aus Syrien). Dabei werden Kontexte erlebter Diskriminierung benannt, die eher im bürokratisch-administrativen Bereich liegen als im sonstigen Alltag. Hingegen wird keine grundsätzliche Dichotomie zwischen unterschiedlichen Kulturen der Herkunftsund Aufnahmegesellschaft erlebt oder konstruiert; auch sind Konstrukte eines typischen „Deutschseins“ im Kontext der geführten Gespräche nicht explizit nachzuweisen.

\section{e) Wahrgenommene Bedeutung des räumlichen Umfelds}

In Nordrhein-Westfalen sind Geflüchtete durch die Wohnsitzauflage in der Wahl ihres Wohnstandortes eingeschränkt; sie dürfen nur innerhalb der ihnen zugewiesenen Stadt oder Gemeinde umziehen. Insofern spielten Aussagen über das für ein Sich-Zuhause-Fühlen ideale räumliche Umfeld eine untergeordnete Rolle. Auch innerhalb der Gemeinde bestimmten nicht die Wünsche der Geflüchteten den Wohnstandort, sondern der Wohnungsmarkt. Die Knappheit an geeignetem Wohnraum führt dazu, dass außerhalb der Heime jede Wohnung, die angeboten (und vom Jobcenter bzw. Sozialamt bezahlt) wird, akzeptiert werden muss. Die verschiedenen Wege der Wohnungsvermittlung führen dazu, dass, jenseits von gelegentlich aus Kostengründen gebildete Wohngemeinschaften, keine lokale Konzentration von Geflüchteten oder bestimmten ethnischen Gruppen auftritt: Da die Vermittlung über städtische Wohnbaugesellschaften, das Sozi- alamt, die Kirche, Ehrenamtler oder professionelle private VermieterInnen erfolgt, entsteht Nähe zu Verwandten oder Bekannten eher zufällig. So wird oft erst im Nachhinein entdeckt, dass andere Personen der gleichen Nationalität oder ein Freund aus dem Heimatort in derselben Straße wohnen. Niemand deutet an, für seine Wohnumgebung eine ethnische Gemeinschaft mit Landsleuten anzustreben. Wer auf bestimmte, im deutschen Standard-Einzelhandel nicht erhältliche Waren aus seiner Herkunftsregion Wert legt, kann solche Anbieter auch mit öffentlichen Verkehrsmitteln erreichen (z.B. arabische/persische Läden im Stadtteil Köln-Kalk).

Eine Bewertung des Wohnstandortes im Hinblick auf soziale Kontakte geschieht in der Regel in der Dichotomie „,(Groß-)Stadt“ versus „Land“. Nicht nur GesprächspartnerInnen im Kreis Heinsberg, sondern auch manche in Köln äußern die Überzeugung, dass man sich in einem Dorf wohler fühlt im Sinn von ,auf dem Land ist das Leben einfacher“, „man lernt dort schneller neue Leute kennen“, „die Menschen sind tendenziell freundlicher". „Ich hatte viele Hilfe von Leuten, netten Leuten. Auch die Hilfe vom Sozialamt war ok. Und es ist hier in Hückelhoven in einer Kleinstadt auch besser als in einer Großstadt. Ich glaube zu lernen und zu leben, das Leben ist einfacher, glaube ich" (Herr C, 25 Jahre, Heinsberg, aus Syrien). Andere Geflüchtete präferieren wiederum ein städtisches Umfeld, da es mehr berufliche Optionen bietet bzw. bei einem geplanten Studium aufgesucht werden müsste. Als ein weiterer Vorteil wird die großstädtische Diversität wahrgenommen. „To add something, the greatest thing in Cologne its diversity, you find people from all nationalities [...] Here in Cologne you find a lot of nationalities, and they are very cooperative with Germans as if they are really Germans, as if they were born here." (Frau E, 21 Jahre, Köln, aus Syrien). Die positiv bewertete Multinationalität bedeutet hier weniger die Chance, auf Landsleute zu treffen, sondern die Aufnahmegesellschaft als ethnischkulturelles Kontinuum zu erleben, im Sinn einer Vorstellung darüber, dass Personen verschiedener Herkunft miteinander und mit den alteingesessenen Deutschen friedlich zusammenleben und kooperieren können.

\subsection{Subjektive Integrationsvorstellungen von Personen, die beruflich oder ehrenamtlich in der Betreuung von Geflüchteten tätig sind}

Wie bei den GesprächspartnerInnen mit Fluchterfahrung, so konnten auch aus den Aussagen der AkteurInnen aus der Betreuung von Geflüchteten typische Elemente einer subjektiven Theorie der Integration von FluchtmigrantInnen abgeleitet werden.

\section{a) Grundlegende Voraussetzungen seitens der Geflüchteten}

Auch unter den GesprächspartnerInnen aus der beruflichen oder ehrenamtlichen Hilfe für Geflüchtete besteht im We- 
sentlichen Konsens über einige zentrale Merkmale einer „Integration" in die Aufnahmegesellschaft: Das Beherrschen der deutschen Sprache, eine eigene Wohnung als Privatsphäre, eigenes Einkommen aus qualifizierter Erwerbsarbeit und die Präsenz der eigenen Kernfamilie. Bestandteil dieses Konsenses ist auch, dass diese Voraussetzungen nicht etwas sind, das die Geflüchteten einseitig zu erbringen haben. Sie werden vielmehr als ein Resultat von Angeboten der Aufnahmegesellschaft (Sprachkurse, ausreichend preisgünstiger Wohnraum, berufliche Qualifikationsmaßnahmen, geeignete Ausbildungs- und Arbeitsplätze) und der Bereitschaft der MigrantInnen, diese anzunehmen, betrachtet. Der Fokus auf Privatsphäre und Familienzusammenführung zeigt, dass die HelferInnen diese Probleme der Geflüchteten durchaus zutreffend erkannt haben. Die Bereitschaft zu wohnen und zu arbeiten, wird nicht als Anpassungsleistung gewertet.

Nötige und offenbar nicht selbstverständliche Anpassungen der Geflüchteten werden im Bereich des Bildungssystems, der Sprache und der Alltagspraxis gefordert. Das Spezifikum des deutschen Systems der schulischen und beruflichen Ausbildung wird in gegenüber manchen Herkunftsländern höheren Anforderungen gesehen. Hier heben die GesprächspartnerInnen hervor, dass viele Berufe nicht als Folge bloßen Anlernens ausgeübt werden können, sondern qualifizierender Abschlüsse im dualen System bedürfen. Auch die Schulpflicht für Mädchen wie Jungen wird als nicht diskutable Gegebenheit gesehen, der sich die Menschen mit Fluchterfahrungen zu stellen haben. Dies gilt ebenso für Spracherwartungen, die für bestimmte Qualifikationen (z.B. Abitur, Zulassung zum Studium) vorgegebene, durch Prüfungen und Zertifikate nachzuweisende Normen des europäischen Referenzrahmens für Sprachen (A1-C2) voraussetzen, so dass eine im Alltag gelernte und vielleicht dort auch praxistaugliche Sprachbeherrschung nicht genügt.

Anpassung erwartet wird auch an grundlegende Regeln der deutschen Alltagspraxis, zum Beispiel die Funktionsweise der Bürokratie, das regelkonforme Benutzen des öffentlichen Nahverkehrs, aber auch der Umgang mit Gefahren, wie Verschuldung über Handyverträge, oder Aufnehmen schlechter Arbeit für schnelles Geld. Grundsätzlich wird jedoch eine gegenseitige Wertschätzung und Akzeptanz ohne einseitige Anpassung der Zuwanderer gefordert. Ähnlich gemeinte Formulierungen sind Begegnung auf Augenhöhe, miteinander lernen oder interkulturelle Sensibilität. So heben die GesprächspartnerInnen z.B. hervor, dass das Erlernen der deutschen Sprache keine Abkehr von der Muttersprache bedeuten soll, sondern Mehrsprachigkeit vielmehr als Ressource zu verstehen ist, die es zu fördern gilt. Ein Beibehalten der eigenen Sprache (Deutsch als Zweitsprache), auch bei den Kindern, sowie der eigenen religiösen und kulturellen Identität gilt als selbstverständlich. Statt einer unterstellten Homogenität wird die Mischung der Gesellschaft als Grundvoraussetzung der Integration betrachtet. In Ansätzen lassen die Gespräche auch Elemente traditioneller Vorstellungen der Anpassung an den kulturellen Mainstream im Sinne einer als be- sonders gelungen empfundenen Integration erkennen. Diese werden z.B. wahrgenommen, wenn Freundschaften mit Einheimischen geschlossen werden, man sich an üblichen Einsätzen für die „Gemeinschaft“ beteiligt (z.B. abwechselnd Kinder eines Freundeskreises zur Schule bringen) und lokalregionale Kulturelemente aktiv übernommen werden, sei es der Straßenkarneval oder das Martinsbrauchtum: , [...] [D]ie haben dann alle eine Fackel bekommen und konnten dann auch am Sankt-Martins-Zug teilnehmen. Also so, das ist Integration live sag ich mal. So, das ist das Beispiel, wie man vorgehen muss [...] Wir wollen, dass sie unsere Kultur verstehen und auch mitmachen " (Herr H, Heinsberg).

Die Geflüchteten werden von den AkteurInnen der Betreuung von Geflüchteten nicht primär nach Herkunftsländern oder Kulturkreisen differenziert und typisiert. Dennoch werden die FluchtmigrantInnen in Gruppen eingeteilt, die einer unterschiedlichen Behandlung bedürfen oder deren Integration in Bildungssystem sowie Arbeitsmarkt mit unterschiedlichen Schwierigkeiten behaftet ist. Nach dem Kriterium der gesetzlichen Vorgaben erfolgt eine Unterscheidung von Gruppen unterschiedlicher Aufenthaltsberechtigung, welche sich häufig an Nationalitäten politisch festgelegter unsicherer versus sicherer Länder orientiert. Der Aufenthaltsstatus wiederum ist wichtig für den Zugang zu einzelnen Unterstützungsleistungen (z.B. Integrations- und Sprachkurs) und Zuständigkeiten (z.B. Sozialamt oder Jobcenter). Nach dem Kriterium des Bildungsgrades werden Geflüchtete ohne Alphabetisierung, mit arabischer oder arabischer und englischer Alphabetisierung sowie nach vorhandenen Bildungsabschlüssen unterschieden. Gerade die unterschiedliche Alphabetisierung stellt besondere Ansprüche an die Beschaffenheit der Sprachkurse. Beim Umgang mit dem Grad der Vorbildung schimmern in den Aussagen der in der Hilfe für Geflüchtete Tätigen ansatzweise Typisierungen nach Herkunft (arabisch versus afrikanisch) durch. Dabei besteht grundsätzlich eine gewisse Tendenz, die Ausbildung der Geflüchteten pauschal als vormodern, landwirtschaftlich bzw. traditionell handwerklich geprägt zu interpretieren: „Tourismus kennen die dann im Zweifelsfall nicht, denn wer kommt schon in die syrische Steppe oder so" (Herr K, Heinsberg). Ebenso wird die kulturelle Prägung der NewcomerInnen in der Tendenz als patriarchalisch mit struktureller Benachteiligung der Frauen dargestellt. Das Kriterium des Alters ist relevant für die Platzierung im (Aus-)Bildungssystem entsprechend der folgenden Fragen: Greift noch die Schulpflicht, ist das Nachholen von Abschlüssen möglich oder muss direkt eine Überführung in Ausbildungsverhältnisse angestrebt werden? Gängig ist zudem bei den ehrenamtlich tätigen GesprächspartnerInnen eine implizite oder explizite Unterscheidung zwischen aktiven, engagierten Leuten, die sich helfen lassen und passiven, für Angebote nicht erreichbare Personen. 
b) Gesellschaftliche Maßnahmen der

Integrationsförderung

Als zentrales Element einer politisch-administrativen Integrationsförderung wird die Bildungskette gesehen. Dem Faktor Bildung wird von den in der Hilfe für Geflüchtete tätigen GesprächspartnerInnen die Schlüsselrolle im Zugang zu gesellschaftlicher Teilhabe zugesprochen. Um Geflüchteten eine reale Chance auf dem Arbeitsmarkt zu geben, soll ihnen, in Abhängigkeit von Alter und Kenntnissen, ein adäquater Platz im (Aus-)Bildungssystem, beginnend mit vorschulischer Betreuung, vermittelt werden. Die Übernahme unqualifizierter Tätigkeiten durch Geflüchtete (,Jobs“) wird als Zwischenlösung zum Aufbessern des Einkommens gesehen, aber nicht als eine langfristige Lösung betrachtet. Im Hinblick auf zeitliche Horizonte wird anerkannt, dass Integration Zeit benötigt; anderseits wird aber auch betont, dass sich staatliche Integrationsförderung nicht zu viel Zeit lassen sollte, da immer wieder neue FluchtmigrantInnen einreisen. Diese Förderung wird daher von den GesprächspartnerInnen als permanente Aufgabe verstanden, die nicht irgendwann einmal aufhört und daher einer gewissen Effizienz bedarf. Die Vorstellung einer Normalität von Integrationsförderung kommt auch in der von einigen GesprächspartnerInnen geäußerten Wahrnehmung der Geflüchteten als „Kunden der Bürokratie“ zum Ausdruck.

\section{c) Wahrgenommene gesellschaftliche Rahmenbedingungen}

Die Integrationsbereitschaft der Bevölkerung wird von den AkteurInnen der Betreuung von Geflüchteten berücksichtigt, allerdings nicht an zentraler Stelle und hinter den politischadministrativen Maßnahmen rangierend. Die ehrenamtlich, aber auch die beruflich Geflüchtete Betreuenden beklagen nationalstaatliche bürokratische Vorgaben als Hindernisse bei einer effektiven Eingliederung der Geflüchteten in die Aufnahmegesellschaft. Dazu zählen die Kategorisierung von Herkunftsländern nach Sicherheit, Zuweisungspraktiken, unterschiedliche und wechselnde Zuständigkeit, z.B. Wechsel von Asylsuchenden nach Anerkennung vom Sozialamt zum Jobcenter, Vorbedingungen für Eröffnen eines Girokontos, mangelnde Abstimmung von Integrationskursen mit Jobzeiten, etc. Eine gewisse Fremdenfeindlichkeit bzw. Alltagsrassismus in der Gesellschaft wird aus Sicht der HelferInnen konstatiert (z.B. an Stammtischen oder bei Empfängern staatlicher Transferleistungen) - diese Problematik wird jedoch nicht in einem Ausmaß wahrgenommen, das die Integration der Zuwandernden essenziell behindern könnte, auch wenn sich im Alltagsdiskurs nach den Kölner ,Sylvesterereignissen“ 2016/17 eine Distanzierung von Geflüchteten, gerade aus arabischen Ländern, bemerkbar gemacht hat. Einzig die Diskriminierung durch Vermieter wird beklagt. Dies wird von den Befragten allerdings weniger auf rassistische Haltungen, sondern auf die kurzen Mietverträge und unsichere
Mietzahlungen zurückgeführt. Ehrenamtlich Tätige betrachten ihre eigene Arbeit im Allgemeinen positiv und werden auch von AkteurInnen der administrativen Betreuung von Geflüchteten als wichtige, für die Belange der Geflüchteten offene Helfer, geschätzt.

Einige wenige GesprächspartnerInnen stellen die Arbeitsmarktintegration der Geflüchteten auch in den Kontext gesellschaftlicher Trends, allerdings bei widersprüchlichen Deutungen dieser Entwicklungen. Zum einen wird die optimistische Auffassung vertreten, dass in Deutschland alle Altersgruppen und Qualifikationen in eine passende berufliche Tätigkeit vermittelbar sind; auch mit 50 Jahren ist es in Deutschland noch möglich, sich beruflich zu verändern. Begünstigt wird diese Sicht durch die aktuelle Wirtschaftslage mit niedriger Arbeitslosigkeit und Facharbeitermangel. Dem steht die eher pessimistische Perspektive gegenüber, die auf den generellen Trend, menschliche Arbeit durch Maschinen zu ersetzen, fokussiert. Demnach können Menschen über 50 nicht mehr in ein Arbeitsverhältnis vermittelt werden; diesen Zuwanderern sollten Perspektiven eines sinnvollen Lebens jenseits von Erwerbstätigkeit aufgezeigt werden, statt sie zu diskriminieren.

\section{d) Abgrenzung vom „offiziellen“ Integrationsbegriff}

Einige GesprächspartnerInnen thematisieren den Begriff der „Integration“ als Ausdruck von Ideologie und theoretischen Ansätzen. In einer kritischen Sicht wird aktuelle Integrationspolitik als eigentlich ausgrenzend verstanden, da sie mit der Differenzierung in sichere versus unsichere Länder sowie in Asylberechtigung und subsidiären Schutz Personen unterschiedlichen Bleiberechts schafft, denen Türen geöffnet werden oder eben nicht. Weiterhin wird Integration als Vorstufe der Inklusion in einer Abstufung des gesellschaftlichen Umgangs mit Diversität verstanden, wobei Inklusion die höchste Stufe darstellt. Einem „offiziellen“ politisch-ideologischen Verständnis stellen die in der Flüchtlingshilfe Tätigen eigene Definitionen gegenüber. So beispielsweise ein Verständnis von Integration als Gefühl oder als Einladung, die Gesellschaft mitzugestalten ohne Verpflichtung zur Anpassung: „Ich finde, keiner muss sich integrieren. Ist so, Ich find, das ist in einem demokratischen, freien Staat, es gibt doch keine Pflicht. [...] Die Gesellschaft muss aushalten, dass manche keine Lust auf sie haben. " (Frau EK, Köln). Dieses liberale Verständnis geht einher mit einer Kritik an der geltenden Gesetzeslage „Wenn du den Weg zu uns findest, gibt es eine Chance für dich, wenn du dich bewährst, dass du immer mehr zu uns gehören kannst. Aber dafür musst du ganz viele Voraussetzungen erfüllen. So läuft das ja. Das heißt, das Ausländerrecht ist sozusagen das Gegenteil von Integration" (Herr P, Köln). Neben einer überzeugten kritischen Haltung ist auch eine eher diffuse Unsicherheit gegenüber einem Integrationsbegriff anzutreffen: ,Aber ich weiß immer nicht, $o b$ das bedeutet, dass er integriert ist, weil wenn er mit den Nachbarn links und rechts hat keinen Bock zu reden und 
trotzdem, sag ich mal, nur mit seinen syrischen oder eriträischen Kumpels irgendwo trifft, ich kann diese Frage [...] ich weiß es nicht." (Frau W, Heinsberg).

\section{e) Bewertung der räumlichen Verteilung der Geflüchteten und des sozialräumlichen Umfelds}

In einer Art subjektiven „Theorie“ der Dezentralisierung gehen einige der GesprächspartnerInnen davon aus, dass eine disperse Verteilung der Geflüchteten sich günstig auf Integration auswirkt, da leichter Kontakte zu (einheimischen) Nachbarn entstehen, eine größere Kontinuität dieser Kontakte gewährleistet ist und öffentliche Angebote weniger überlastet sind. Bei zentraler Unterbringung wird die Gefahr rein innerethnischer Kontakte gesehen, daraus resultierend eine verminderte Bereitschaft zu Eingliederung der Geflüchteten einerseits und wachsende Ressentiments der Einheimischen andererseits. „Ich bin ein Gegner von zentraler Wohnungsversorgung, Unterbringung von Flüchtlingen. Das schafft für meine Begriffe gesellschaftliche Polarisation, das schafft Distance, das schafft, ja, Ressentiments im Zugang der etablierten Bevölkerung zu den Flüchtlingen und deshalb ist die dezentrale Wohnungsversorgung vor Ort integrativ" (Herr P, Heinsberg). Gemäß dieser Theorie bringt ein Leben in der ethnischen Kolonie ohne deutsche Sprachkenntnisse mit unqualifizierten Jobs nicht weiter, sondern führt eher in (Drogen-)Kriminalität: ,Klar, ist natürlich super, wenn die ... dann haben die auch ein bisschen Heimatgefühl, weil die sind dann auch Iraker oder Marokkaner oder was. Aber ich find das schwierig zu durchblicken. Ob das jetzt gut ist oder schlecht, weiß man immer nicht so genau und wir haben auch Drogenprobleme“ (Frau K, Köln). Dem gegenüber steht eine liberalere Sichtweise, die den Zuwanderern zugesteht, unter sich zu bleiben, wenn sie dies beabsichtigen. So wird zum Beispiel auf die Europäische Menschenrechtskonvention verwiesen, die zwar den Schutz der Privatsphäre, nicht aber Kontakte zur Aufnahmegesellschaft zwingend vorsieht. Kontakte vorwiegend innerhalb der eigenen ethnischen Gemeinschaft in segregierten Quartieren wären demnach zu tolerieren. „Ansonsten finde ich das ja auch überhaupt gar nicht schlimm. Also wenn das eine selbst gewählte Gemeinschaft ist, dass man da Zentren hat [...] wenn die Menschen auch eine Chance haben, sich anders zu entscheiden und das nicht auf Missständen beruht" (Frau KB, Köln). Diese liberale Sichtweise rekurriert auf eigene, bislang positive Erfahrungen mit ethnisch geprägten Quartieren, wie im folgenden Zitat zum Beispiel mit Bezug auf die Stadt Köln: ,,[...] da ist eine Keupstraße und da kann man super türkische Lebensmittel einkaufen und gut essen gehen, das finde ich überhaupt nicht schlimm [...]" (Frau KB, Köln).

Eine subjektive Theorie spezifischer dörflicher Integrationskontexte wird von GesprächspartnerInnen konstruiert, die im ländlich-kleinstädtischen Bereich tätig sind. Dem eher dörflichen Umfeld werden Mängel bei der Versorgung mit besonders großen und kleinen Wohnungen sowie Defizite bei wohnortnahen bzw. per öffentlichem Nahverkehr gut erreichbaren Bildungs- und Arbeitsangebot als das Einleben bzw. den Alltag erschwerende Eigenschaften attestiert. Die Einschätzung der sozialen Integrationsqualitäten fällt dagegen uneinheitlich aus: Einerseits werden Befunde einer größeren Verbindlichkeit und Herzlichkeit gegenüber Geflüchteten betont. Anderseits wird das dörfliche Umfeld als konservativ erlebt, im Sinne eines stärkeren Bezugs auf kulturelle Traditionen und geringere Toleranz. „Ob es dann um das Brauchtum bei den Schützen darum geht, ne, wie katholisch muss man sein, um Schützenkönig zu sein, und darf man - darf 'n Mann 'nen Mann als Freund haben, das kann man in der Stadt alles - ist das - spielt das tendenziell schon keine Rolle mehr. Auf'm Dorf wird das immer noch mal ganz anders diskutiert [...].“ (Herr K, Heinsberg).

Vorstellungen über raumbezogene Einflüsse auf das Einleben beziehen sich aber auch auf die Mikroebene des Wohngebäudes, wenn von einigen GesprächspartnerInnen gefordert wird, Belegung mit Geflüchteten verschiedener Herkunft oder Religion aufgrund der drohenden Konflikte zu vermeiden.

\section{Fazit: Subjektive Integrationsvorstellungen - Auf dem Weg in postmigrantische Stadtgesellschaften?}

Zusammenfassend kann gesagt werden, dass sowohl in den Äußerungen der befragten Geflüchteten als auch der in der Betreuung von Geflüchteten Tätigen zum Teil durchaus postmigrantische Vorstellung von Integration aufscheinen. Und zwar in dem Sinn, dass eine starke und durchgängige Prägung der deutschen Gesellschaft durch Migration von allen GesprächspartnerInnen anerkannt und für selbstverständlich gehalten wird (Foroutan et al., 2014; Foroutan, 2015). Dieser Befund lässt vermuten, dass sich Institutionen, politische und nicht zuletzt auch subjektive Vorstellungen der erkannten Migrationsrealität schrittweise angepasst haben.

Für die Geflüchteten bedeutet ein Sich-Zuhause-Fühlen in der Aufnahmegesellschaft eindeutig gesellschaftliche Teilhabe unabhängig vom Migrationshintergrund, zuvorderst orientiert an den Grundbedürfnissen Familie, Sicherheit, Arbeit und Wohnen. Nationale Identitäten spielen in den Aussagen eine untergeordnete Rolle, so ließen die Gespräche kaum dichotome Bezüge auf unterschiedliche Kulturen der Herkunfts- und Aufnahmegesellschaft erkennen. Die deutsche Gesellschaft erscheint nicht als monolithische Leitkultur, in die hinein sich Newcomer unter Aufgabe ihrer alten kulturellen Identität zu integrieren haben, sondern eher als ein kulturell-ethnisches Kontinuum, in dem es teilweise schwer ist, zu erkennen, bei wem es sich eigentlich um einen ,echten“ Deutschen handelt. Insofern erkennen auch die Geflüchteten implizit aus ihrer Alltagserfahrung heraus an, dass Deutschland eine von Zuwanderung geprägte Gesellschaft ist. Sprache wird nicht als Element von Kultur be- 
griffen, sondern als funktionaler Schlüssel, der in einer dominant anderssprachigen Gesellschaft notwendig ist, um das angestrebte normale Leben führen zu können. Teilhabe wird fokussiert auf den ökonomischen Bereich der Gesellschaft; politische Rechte und Fragen einer Staatsbürgerschaft werden (noch) nicht thematisiert. Für Rechtsgleichheit und Diskriminierungen im Alltag besteht jedoch ein feines Gespür, empfundene Missstände werden deutlich angesprochen.

Auch die beruflich oder ehrenamtlich in der Hilfe für Geflüchtete Tätigen bauen in der Beschreibung der Merkmale von Geflüchteten und der Aufnahmegesellschaft keine dichotomen Kulturstereotype auf, in denen vermeintlich typisch deutsche den typischen Merkmalen der Herkunftsländer gegenübergestellt werden. Vielmehr wird die individuelle Situation der Geflüchteten nach administrativen Kriterien wie amtliche Sicherheitskategorie des Herkunftslands, Schutzstatus, schulpflichtiges Alter, Alphabetisierungsgrad etc. differenziert. Differenzierungen in strukturell begünstigte Geflüchtete, die höhere Bleibechancen und eine bessere Bildung haben, und dem gegenüber Benachteiligte, sind feststellbar, aber nicht exkludierend gemeint: Weniger gebildete Geflüchtete stellen die deutsche Gesellschaft vor größere Herausforderungen, sind aber nicht abzulehnen. Dennoch ist eine subtile paternalistische Haltung anzutreffen, die betont, was für das Einleben in Deutschland zwar nicht kulturell, aber funktional notwendig ist. Integration im Verständnis der in der Hilfe für Geflüchtete Tätigen bedeutet dabei keine Anpassung an eine deutsche Leitkultur, aber an die Anforderungen einer „modernen“ (europäischen) Gesellschaft, wobei in Ansätzen durchscheint, dass die mitgebrachten Kompetenzen und Werte nicht immer mit diesen Anforderungen korrespondieren (siehe Kap. 4.2 a). Manche InterviewpartnerInnen empfinden die Betreuung Geflüchteter daher als ,,pädagogische Arbeit", bei der die Zugewanderten in die Notwendigkeiten des deutschen Systems (z.B. Verbindlichkeit, Eigenverantwortung, realistische Forderungen) hinein zu erziehen sind. Integration wird übereinstimmend verstanden als die Chance, Grundbedürfnisse zu befriedigen sowie den Alltag im neuen Umfeld selbständig bewältigen zu können. Zentrale Bausteine sind dabei - durchaus ähnlich den Vorstellungen der Menschen mit Fluchterfahrung - das Beherrschen der deutschen Sprache, eine eigene Wohnung, eine (qualifizierte) Arbeitsstelle sowie die eigene Familie vor Ort (ähnliche Ergebnisse siehe z.B. Nestvogel, 2014).

Die Sichtweisen unterscheiden sich kaum zwischen den AkteurInnen in der Großstadt Köln und des klein- und mittelstädtisch geprägten Kreises Heinsberg bis auf die stärker erkennbare paternalistische Tendenz der HeinsbergerInnen. Dies deutet einerseits darauf hin, dass nicht nur in großen urbanen Agglomerationen, sondern auch in kleinen Städten migrationsgesellschaftliche Bedingungen zunehmend in institutionellen als auch alltagsweltlichen Kontexten angekommen sind und anerkannt werden. Einerseits sind hier die langjährigen lokalen Zuwanderungserfahrungen von Bedeutung, wie im Fallbeispiel Heinsberg die Vorgeschichte der Gastar- beiterzuwanderung. Andererseits lassen diese Befunde sich unter Umständen auch auf den gemeinsamen, spezifischen und dominanten Bezugsrahmen „Fluchtmigration“ zurückführen, der im Alltag für beide Gruppen in beiden räumlichen Kontexten zentral und daher mit einer spezifischen Sicht auf Gesellschaft verknüpft ist. Denn im Unterschied zu den vorliegenden Ergebnissen konnten für Befragte in anderen Studien eher Perspektiven auf Integration im Sinn der Assimilation und einseitigen Anpassung (Zick und Preuß, 2016) bzw. ein Vorherrschen traditioneller dichotomischer Vorstellungen von einheimisch und fremd aufgezeigt werden (z.B. Foroutan et al., 2014), wonach Deutschsein zwar erlernt werden kann, aber immer noch exklusive Elemente besitzt, wie ein akzentfreies Beherrschen der deutschen Sprache.

Keine Gruppe der GeprächspartnerInnen benennt eine Liste von für eine vollständige Integration notwendigen Parametern oder Konstrukte eines typischen Deutschseins. Dagegen betonen beide den Anspruch der Geflüchteten auf gesellschaftliche und staatliche Unterstützung beim Einleben und identifizieren vor allem bürokratischen Regelungen als relevante Barrieren des Einlebens.

In ihren Vorstellungen über Räume des Sich-ZuhauseFühlens greifen weder Geflüchtete noch AkteurInnen der Betreuung von Geflüchteten auf das Konzept der ethnischen Kolonie zurück. Eine ethnische Konzentration in bestimmten Quartieren wird von keiner Seite gezielt angestrebt, das Grundbedürfnis des Wohnens kann an jedem Standort befriedigt werden. Im Hinblick auf das sozialräumliche Umfeld ihrer Wohnung sind die Geflüchteten pragmatischer; der Diskurs um soziale Mischung geht an ihren subjektiven Anforderungen und Realitäten vorbei. Relevant sind für die GesprächspartnerInnen mit Fluchterfahrung vielmehr funktionale Gesichtspunkte der Nähe und Lage des Wohnstandortes: die Wege zum Integrations- und Sprachkurs, zur Schule oder Kindertagesstätte der Kinder, sowie auch zu Praktikums/Ausbildungs- oder Arbeitsplätzen sind vor allem bedeutsam, da Geflüchtete auf den öffentlichen Nahverkehr angewiesen sind. Dem gegenüber reflektieren AkteurInnen der Geflüchtetenhilfe verstärkt disperse versus konzentrierte Verteilungen der Zuwanderer auf Gebäude-, Gemeinde- und vor allem auf Quartiersebene im Hinblick auf Integration fördernde Bedingungen. Dabei erweist sich die Kontakthypothese in den Köpfen als eine wichtige und dominierende Vorstellung: Soziale Mischung von Zugewanderten und „Einheimischen“ wird begrüßt, die residenzielle Segregation von Geflüchteten als „Ghettoisierung“ abgelehnt - eine Vorstellung, die auch weitestgehend dem dominierenden öffentlichen Diskurs entspricht (z.B. Heitmeyer, 1998). Teilweise wird jedoch auch die freiwillige residenzielle Segregation als zu akzeptierendes Recht der Zugewanderten postuliert.

Im klein- und mittelstädtisch geprägten Kreis Heinsberg tendieren sowohl die Geflüchteten als auch die BetreuerInnen zudem eher dazu, die Vor- und Nachteile eines Wohnstandorts in einer Großstadt gegenüber einem eher ländlichdörflichen Raum abzuwägen. Dabei wird die dörfliche Über- 
schaubarkeit in sozialer Hinsicht von beiden Gruppen tendenziell positiv bewertet.

Unter Rückbezug auf die Überlegungen zur räumlichen Maßstabsebene (vgl. Kap. 2) kann gesagt werden, dass sowohl aus der Sicht von Geflüchteten als auch von ihren BetreuerInnen das Einleben auf der nationalstaatlichen Ebene Deutschlands von gesetzlichen Vorgaben beeinflusst wird. Transnationale Bezüge zum Herkunftsland werden von den BetreuerInnen zum Teil eher als Barriere für das Einleben wahrgenommen, wenn dort noch Teile der Kernfamilie leben und die dort erworbenen Qualifikationen für den deutschen Arbeitsmarkt unzureichend sind. Für die Geflüchteten besitzt ihr Herkunftsland neben der Sorge um noch dort lebende Familienmitglieder auch emotionale biographische Bezüge. Zudem arbeiten beide Seiten auf der regionalen Ebene mit einem Stadt-Land-Gegensatz. Während die Quartiersebene unter dem Aspekt der ethnischen Segregation bzw. der sozialen Mischung nur in den Augen der BetreuerInnen Relevanz besitzt, steht die Bedeutung eines privaten Refugiums auf der Mikroebene insbesondere in Form einer eigenen Wohnung für alle GesprächspartnerInnen außer Zweifel.

Inwiefern die Wohnstandorte der Geflüchteten sich nach Ende der Frist für den Wohnsitzzwang verändern, kann derzeit kaum vorausgesagt werden. Offen bleibt auch die Frage, inwieweit und in welcher Form sich die neuen Alltagskontexte und -routinen der Geflüchteten zu neuen Transtopien aus Herkunfts- und Ankunftsräumen verdichten werden (Yildiz, 2017:24). Phänomene eines Ankommens auf eigene Rechnung durch ökonomische Selbständigkeit sind nur am Rande als vage Zielvorstellungen zu identifizieren. Die meisten der jüngeren befragten Geflüchteten streben eine formale Ausbildung (beruflich, universitär) an; diese würde den Übergang in den normalen Arbeitsmarkt in abhängiger Beschäftigung erlauben. Mit dem Gedanken an Selbständigkeit spielen nur wenige, ältere Befragte mit Fluchterfahrung (z.B. Eröffnung eines iranischen Restaurants, Eröffnung einer eigenen Schneiderei).

Die Ergebnisse deuten an, dass sich die Praxis des Ankommens sowohl für die Geflüchteten als auch für die auf lokaler Ebene im Bereich der Geflüchtetenhilfe Tätigen - ob behördlich oder ehrenamtlich - anders darstellt, als in der nationalen öffentlich-politischen Debatte. Beide Gruppen sind sich in der Sicht wesentlicher Elemente und Notwendigkeiten des Einlebens in die Ankunftsgesellschaft einig. Insofern scheint die Realität einer postmigrantisch-pragmatischen Sicht auf Integration im alltäglichen Miteinander von Menschen mit Fluchterfahrung und Menschen, die in der Geflüchtetenhilfe tätig sind, trotz zum Teil erkennbarer, paternalistischer Vorstellung über funktional notwendige Systemanpassungen, der politischen Integrationsdebatte deutlich voraus.

Datenverfügbarkeit. Die empirische Grundlage dieses Beitrages bilden leitfadengestützte Interviews, die sensible, zum Teil personenbezogene Daten enthalten. Da den InterviewpartnerIn- nen Anonymität und Vertraulichkeit zugesichert wurden, sind die Transkripte nicht öffentlich zugänglich. Informationen zum Datenmaterial können beim Corresponding Author erfragt werden.

Autorenmitwirkung. Der Artikel wurde im Wesentlichen vom Corresponding Author verfasst. Die Konzeption des Forschungsprojekts sowie die Erhebung der Daten wurde jedoch von allen genannten AutorInnen gemeinsam durchgeführt.

Interessenkonflikt. Die Autoren erklären, dass kein Interessenkonflikt besteht.

Danksagung. Unser Dank gilt allen InterviewpartnerInnen und dem Forschungsinstitut für gesellschaftliche Weiterentwickung (e.V.) für die finanzille Förderung des dem Artikel zugrunde liegenden Forschungsprojekts „Integration Geflüchteter in groß- und kleinstädtischen Räumen in Nordrhein-Westfalen“. Den GutachterInnen sowie Karin Wiest danke ich außerdem für ihre konstruktiven Überarbeitungshinweise.

Begutachtung. This paper was edited by Karin Wiest and reviewed by two anonymous referees.

\section{Literatur}

Abramson, H. J.: Assimilation and pluralism, in: Harvard Encyclopedia of American ethnic groups, Herausgeber: Thernstrom, S., Orlov, A. und Handlin, O., Harvard University Press, Cambridge, 150-160, 1980.

Adam, F., Föbker, S., Imani, D., Pfaffenbach, C., Weiss, G., and Wiegandt, C.-C.: Social contacts and networks of refugees in the arrival context. Manifestations in a large city and in selected small and medium-sized towns, Erdkunde, 73, 31-45, 2019.

Ager, A. and Strang, A.: Understanding Integration: A Conceptual Framework, J. Refug. Stud., 21, 166-191, 2008.

Alba, R. and Nee, V.: Remaking the American mainstream. Assimilation theory for a new era of immigration, Harvard University Press, Cambridge, London, 2003.

Aumüller, J. und Bretl, C.: Lokale Gesellschaften und Flüchtlinge: Förderung von sozialer Integration, Berliner Institut für vergleichende Sozialforschung, Berlin, http://www.desi-sozialforschung-berlin.de/wp-content/uploads/ kommunale-integration-von-fluechtlingen.pdf (letzter Zugriff: 28.10.2017), 2008.

Aumüller, J., Daphi, P. und Biesenkamp, C.: Die Aufnahme von Flüchtlingen in den Bundesländern und Kommunen. Behördliche Praxis und zivilgesellschaftliches Engagement, Robert Bosch Stiftung, http://wiki.fhpi.de/_media/bosch_studie_ aufnahme_fluechtlinge_2015.pdf (letzter Zugriff: 28.10.2017), 2015.

Bauböck, R.: The integration of immigrants, Institut für Höhere Studien, Wien, 1995. 
Blank, C.: Soziale Integration als Grundlage lokaler Identifikation in ländlichen Räumen, Mitteilungen der Fränkischen Geographischen Gesellschaft, 58, 205-226, 2011.

Brenner, N.: Global, fragmented, hierarchical: Henri Lefebvre's geographies of globalization, Public Culture, 24, 135-167, 1997.

Bundesamt für Migration und Flüchtlinge BAMF (Hrsg.): Aktuelle Zahlen zum Asyl, http://www.bamf.de/ SharedDocs/Anlagen/DE/Downloads/Infothek/Statistik/ Asyl/aktuelle-zahlen-zu-asyl-november-2017.pdf?_blob= publicationFile (letzter Zugriff: 14.05.2018), 2017.

Esser, H.: Integration und ethnische Schichtung, Arbeitspapiere Mannheimer Zentrum für Europäische Sozialforschung 40, Mannheim, 2001.

Filep, B.: Interview and translation strategies: coping with multilingual settings and data, Social Geography, 4, 59-70, 2009.

Fincke, G.: Abgehängt, chancenlos, unwillig? Eine empirische Reorientierung von Integrationstheorien zu MigrantInnen der zweiten Generation, Verlag für Sozialwissenschaften, Wiesbaden, 2008.

Foroutan, N.: Die Einheit der Verschiedenen: Integration in der postmigrantischen Gesellschaft, Bundeszentrale für politische Bildung, Kurzdossier 28, 2015.

Foroutan, N., Canan, C., Arnold, S., Schwarze, B., Beigang, S. und Kalkum, D.: Deutschland postmigrantisch I. Gesellschaft, Religion, Identität - Erste Ergebnisse, Berliner Institut für empirische Integrations- und Migrationsforschung (BIM), Berlin, 2014.

Glaser, B. G. und Strauss, A. L.: Die Entdeckung gegenstandsbezogener Theorie: Eine Grundstrategie qualitativer Sozialforschung, in: Qualitative Sozialforschung, Herausgeber: Hopf, C. und Weingarten, E., Klett-Cotta, Stuttgart, 91-111, 1979.

Glick-Schiller, N. and Caglar, A.: Locating migration. Rescaling cities and migrants, Cornell University Press, Ithaca, 2011.

Göschel, A.: Integration und Stadt, Deutsche Zeitschrift für Kommunalwissenschaften, 1, 5-11, 2001.

Guinan-Bank, V.: Nach Köln: Zwischen Willkommens- und Ablehnungskultur, Bundeszentrale für politische Bildung, Bonn, http://www.bpb.de/politik/extremismus/rechtsextremismus/ 242070/nach-koeln (letzter Zugriff: 14.05.2018), 2017.

Häußermann, H. und Siebel, W.: Soziale Integration und ethnische Schichtung. Zusammenhänge zwischen räumlicher und sozialer Integration, http://www.bmi.bund.de/internet/content/comon/ anlagen/themen/zuwanderung/datenundfakten/haeussermann. pdf (letzter Zugriff: 05.07.2017), 2001.

Heitmeyer, W.: Versagt die "Integrationsmaschine" Stadt? Zum Problem der ethnisch-kulturellen Segregation und ihrer Konfliktfolgen, in: Die Krise der Städte. Analysen zu den Folgen desintegrativer Stadtentwicklungen für das ethnisch-kulturelle Zusammenleben, Herausgeber: Heitmeyer, W., Dollase, R. und Backes, O., Suhrkamp, Frankfurt, 443-467, 1998.

Höcke, C. und Schnur, O.: Integration von Geflüchteten - quo vadis? Diskussionen und Positionen im Spiegel der vhwKommunalumfrage, Forum Wohnen und Stadtentwicklung 4, 201-207, 2016.

Inhetveen, K.: Translation challenges: Qualitative Interviewing in a multi-lingual field, Qualitative Social Research, 8, 28-45, 2012.

Köchling-Farahwaran, J.: Zur Bedeutung von Heimat für ältere Migrantinnen und Migranten, Peter Lang, Berlin u.a., 2019.
Kreis Heinsberg (Hrsg.): Statistisches, https://www. kreis-heinsberg.de/kreisportrait/statistisches/ (letzter Zugriff: 14.05.2018), 2018.

Kuckartz, U.: Einführung in die computergestützte Analyse qualitativer Daten, VS Verlag für Sozialwissenschaften, Wiesbaden, 2010.

Langhoff, S.: Die Herkunft spielt keine Rolle. „Postmigrantisches" Theater im Ballhaus Naunynstraße, Interview geführt von Katharina Donath, http://www.bpb.de/gesellscjagt/kultur/ kulturelle-bildung/60135/interview-mit-shermin-langhoff? $\mathrm{p}=$ all (letzter Zugriff: 23.04.2018), 2011.

Levitt, P. and Waters, M. C.: Introduction, in: The changing face of home. The transnational lives of the second generation, edited by: Levitt, P. und Waters, M. C., Russell Sage Foundation, New York, 1-30, 2002.

Liebig, M.: Das Postmigrantische. Ein neues Konzept für eine kritische Migrationsforschung? Hausarbeit M.A., Berlin, https://www.euroethno.hu-berlin.de/de/forschung/labore/ migration/hausarbeit_postmigrantisch_manuel-liebig.pdf (letzter Zugriff: 23.04.2018), 2015.

Mayring, P.: Qualitative Inhaltsanalyse, Grundlagen und Techniken, Beltz, Weinheim, Basel, 2015.

Nestvogel, R.: Afrikanerinnen in Deutschland. Lebenslagen, Erfahrungen und Erwartungen, Waxmann, Münster, New York, 2014.

Münch, S.: Rahmenbedingungen von Zuwanderung und interkulturellem Zusammenleben in den ostdeutschen Bundesländern eine Bestandsaufnahme, Raumforschung und Raumordnung, 71, 261-271, https://doi.org/10.1007/s13147-013-0215-1, 2013.

Petermann, S.: Persönliche Netzwerke in Stadt und Land, Siedlungsstruktur und soziale Unterstützungsnetzwerke im Raum Halle/Saale, Westdeutscher Verlag, Wiesbaden, 2002.

Porst, L. and Sakdapolrak, P.: How scale matters in translocality: Uses and potentials of scale in translocal research, Erdkunde, 71, 111-126, 2017.

Portes, A.: Conclusion: Towards a new world. The origins and effects of transnational activities, Journal of Ethnic and Racial Studies, 22, 463-477, 1999.

Portes, A. and Rumbaut, R. G.: Immigrant America: a portrait, University of California Press, Berkeley, Los Angeles, CA, 2006.

Reuber, P.: Heimat in der Großstadt. Eine sozialgeographische Studie zu Raumbezug und Entstehung von Ortsbindung am Beispiel Kölns und seiner Stadtviertel, Kölner Geographische Arbeiten 58, Köln, 1993.

Römhild, R.: Jenseits ethnischer Grenzen. Für eine postmigrantische Kultur- und Gesellschaftsforschung, in: Nach der Migration. Postmigrantische Perspektiven jenseits der Parallelgesellschaft, Herausgeber: Yildiz, E. und Hill, M., Transcript, Bielefeld, 37-48, 2015.

Sachs, K.: Ortsbindung von Ausländern. Eine sozialgeographische Untersuchung zur Bedeutung der Großstadt als Heimatraum für ausländische Arbeitnehmer am Beispiel von Köln, Kölner Geographische Arbeiten 60, Köln, 1993.

Saunders, D.: Arrival City, Windmill, London, 2011.

Soeffner, H.-G. und Zifonun, D.: Fußballwelten: Die Ordnung ethnischer Beziehungen, in: Mittendrin im Abseits. Ethnische Gruppenbeziehungen im lokalen Kontext, Herausgeber: Neckel, S. und Soeffner, H.-G., VS Verlag für Sozialwissenschaften, Wiesbaden, 133-162, 2008. 
Spielhaus, R.: Studien in der postmigrantischen Gesellschaft. Eine kritische Auseinandersetzung, in: Kongressdokumentation 4. Bundesfachkongress Interkultur_DIVERCITY_Hamburg 2012, Herausgeber: Kulturbehörde der freien und Hansestadt Hamburg, Hamburg, 96-100, 2014.

Stadt Köln (Hrsg.): Statistisches Jahrbuch 2017, Köln, 2017.

Swyngedouw, E.: Excluding the other: the production of scale and scaled politics, in: Geographies of economics, Herausgeber: Lee, R. und Wills, J., Arnold, London, 167-176, 1997.

Teltemann, J., Dabrowski, S. und Windzio, M.: Ethnische Segregation in deutschen Großstädten. Abschottung oder sozioökonomische Restriktion? Migremus Arbeitspapiere, 1/2013, Bremen, 2013.

Temple, B. and Young, A.: Qualitative Research and Translation Dilemmas, Qual. Res., 4, 161-178, 2004.

Treibel, A.: Migration in modernen Gesellschaften. Soziale Folgen von Einwanderung, Gastarbeit und Flucht, Juventa, Weinheim, München, 2008.

Tsianos, V. S. und Karakayali, J.: Rassismus und Repräsentationspolitik in der postmigrantischen Gesellschaft, Aus Politik und Zeitgeschichte, 13-14/2014, 33-39, 2014.
Weichhart, P., Weiske, G., und Werlen, B.: Place, Identity und Image. Das Beispiel Eisenhüttenstadt, Universität Wien, Institut für Geographie und Raumordnung, Wien, 2006.

Weiss, G.: Heimat vor den Toren der Großstadt. Eine sozialgeographische Studie zu raumbezogener Bindung und Bewertung in Randgebieten des Verdichtungsraumes am Beispiel des Umlandes von Köln, Kölner Geographische Arbeiten 59, Köln, 1993.

Yildiz, E.: Postmigrantische Perspektiven. Aufbruch in eine neue Geschichtlichkeit, in: Nach der Migration. Postmigrantische Perspektiven jenseits der Parallelgesellschaft, Herausgeber: Yildiz, E. und Hill, M., Transcript, Bielefeld, 19-36, 2015.

Yildiz, E.: Postmigrantische Perspektiven auf Migration, Stadt und Urbanität, in: Migration, Stadt und Urbanität. Perspektiven auf die Heterogenität migrantischer Lebenslagen, Herausgeber: Geisen, T., Riegel, C. und Yildiz, E., Springer, Wiesbaden, 19-33, 2017.

Zick, A. und Preuß, M.: ZuGleich. Zugehörigkeit und (Un)Gleichwertigkeit, Kurzbericht zum Projekt, Universität Bielefeld, Institut für interdisziplinäre Konflikt- und Gewaltforschung, Bielefeld, 2016. 